\title{
EVALUATION OF LAYER POTENTIALS CLOSE TO THE BOUNDARY FOR LAPLACE AND HELMHOLTZ PROBLEMS ON ANALYTIC PLANAR DOMAINS
}

\author{
ALEX BARNETT*
}

\begin{abstract}
Boundary integral equations are an efficient and accurate tool for the numerical solution of elliptic boundary value problems. The solution is expressed as a layer potential; however, the error in its evaluation grows large near the boundary if a fixed quadrature rule is used. Firstly, we analyze this error for Laplace's equation with analytic density and the global periodic trapezoid rule, and find an intimate connection to the complexification of the boundary parametrization. Our main result is a simple and efficient scheme for accurate evaluation up to the boundary for single- and double-layer potentials for the Laplace and Helmholtz equations, using surrogate local expansions about centers placed near the boundary. The scheme - which also underlies the recent QBX Nyström quadrature - is exponentially convergent (we prove this in the analytic Laplace case), requires no adaptivity, generalizes simply to three dimensions, and has $O(N)$ complexity when executed via a locally-corrected fast multipole sum. We give an example of high-frequency scattering from an obstacle with perimeter 700 wavelengths, evaluating the solution at $2 \times 10^{5}$ points near the boundary with 11-digit accuracy in 30 seconds in MATLAB on a single CPU core.
\end{abstract}

Key words. potential theory, layer potential, integral equation, Laplace, Helmholtz, scattering

1. Introduction. We are interested in solving boundary-value problems (BVPs) of the type

$$
\begin{aligned}
\left(\Delta+\omega^{2}\right) u & =0 & & \text { in } \Omega \\
u & =f & & \text { on } \partial \Omega
\end{aligned}
$$

where $\Omega \subset \mathbb{R}^{2}$ is either an interior or exterior domain with boundary curve $\partial \Omega$, and either $\omega=0$ (Laplace equation) or $\omega>0$ (Helmholtz equation). We will mostly use the above Dirichlet boundary condition in our examples, and note that Neumann and other types can equally well benefit from our technique. Numerical solution of this type of BVP has numerous applications in electrostatics, equilibrium problems, and acoustic or electromagnetic wave scattering in the frequency domain. The case $f \equiv 0$ includes eigenvalue (cavity resonance) problems for the Laplacian.

The boundary integral approach $[1,24]$ has many advantages over conventional finite element or finite difference discretization of the domain: very few unknowns are needed since the problem is now of lower dimension, provable high-order accuracy is simple to achieve, and, in exterior domains, radiation conditions are automatically enforced without the use of artificial boundaries. Thus the approach is especially useful for wave scattering, including at high frequency [10, 9].

The integral equation approach exploits the known fundamental solution for the PDE,

$$
\Phi(x, y)= \begin{cases}\frac{1}{2 \pi} \log \frac{1}{|x-y|}, & \omega=0, \\ \frac{i}{4} H_{0}^{(1)}(\omega|x-y|), & \omega>0 .\end{cases}
$$

For the interior case, which is the simplest, the BVP (1.1)-(1.2) is converted to a boundary integral equation (BIE), which is of the Fredholm second kind,

$$
\left(D-\frac{1}{2} I\right) \tau=f,
$$

where $\tau$ is an unknown density function on $\partial \Omega, I$ is the identity, $D: C(\partial \Omega) \rightarrow C(\partial \Omega)$ is the double-layer operator with kernel $k(x, y)=\partial \Phi(x, y) / \partial n(y)$, and $n(y)$ is the outward normal at $y \in \partial \Omega$. Numerical solution of (1.4), for instance via the Nyström method [24, Ch. 12] [1, Ch. 4]

\footnotetext{
*Department of Mathematics, Dartmouth College, Hanover, NH, 03755, USA
} 
with $N$ quadrature nodes on $\partial \Omega$, results in an approximation to $\tau$ sampled at these nodes, from which one may recover an approximation to $\tau$ on the whole of $\partial \Omega$ by interpolation (e.g. Nyström interpolation). Finally, one can evaluate the approximate BVP solution at any target point $x$ in the domain as the double-layer potential

$$
u(x)=\int_{\partial \Omega} \frac{\partial \Phi(x, y)}{\partial n(y)} \tau(y) d s_{y}, \quad x \in \Omega .
$$

It is convenient, and common practice, to reuse the existing $N$ quadrature nodes underlying the Nyström method to approximate the integral (1.5) - in other words, to skip the interpolation step; we will call this the native evaluation scheme. ${ }^{1}$ It is common wisdom that this gives an accurate solution when $x$ is "far" from $\partial \Omega$, but a very inaccurate one close to $\partial \Omega$, even when the $\tau$ samples themselves are accurate. Fig. 1.1 (a), which will be familiar to anyone who has tested the accuracy of a BIE method, illustrates this: the error of evaluation grows to $O(1)$ as one nears $\partial \Omega$. (Also see [18, Fig. 2] or [21, Fig. 4] which show a similar story for a panel-based underlying quadrature.)

There are several ways to overcome this problem near the boundary. In order of increasing sophistication, these include: i) increase $N$ in the Nyström method (although solving a linear system larger than one needs is clearly a waste of resources); ii) fix $N$, but then interpolate $\tau$ onto a finer fixed set of boundary nodes, enabling points closer to $\partial \Omega$ to be accurately evaluated [1, Fig. 7.4] (this is implemented in [2] - what is not discussed is that the number of finer nodes must grow without limit as $x \rightarrow \partial \Omega$ ); iii) use an adaptive quadrature scheme for (1.5) which is able to access the $\tau$ interpolant [14] (although this achieves high accuracy for each point $x \in \Omega$, we have found it very slow [5, Sec. 5.1] because the adaptivity depends on $x$, with an arbitrarily large number of refinements needed as $x \rightarrow \partial \Omega$ ); iv) use various fixed-order methods based upon precomputed quadratures [26] or grids [25] for the Laplace case; or v) use high-order methods of Helsing-Ojala [18] for the Laplace case that approach machine precision accuracy while maintaining efficiency within a fast multipole (FMM) accelerated scheme.

The method of [18] has recently been extended to the Helmholtz equation in two dimensions [17]. Here we present an alternative, simple, and efficient new method that addresses the closeto-boundary quadrature problem, with numerical effort independent of the distance of $x$ from $\partial \Omega$. One advantage is that our scheme extends naturally to the three-dimensional case, unlike existing high-order two-dimensional schemes. Incidentally, our scheme equally well evaluates the potential on the curve $\partial \Omega$ (i.e. the limit of $x$ approaching $\partial \Omega$ from one side), meaning that it can also be used to construct high-order Nyström quadratures to solve the BIE (1.4) itself; the resulting tool is called QBX [21].

Firstly, in section 2 we analyze (in Theorems 3 and 8) the evaluation error for Laplace double-layer and single-layer potentials, with the global periodic trapezoid rule on an analytic curve $\partial \Omega$ with analytic data - we are surprised not to find these results in the literature. This is crucial in order to determine the annular boundary strip region in which native evaluation is poor; it is within this strip that the new close-evaluation scheme is used. We present the scheme in section 3: simply put, the idea is to interpolate $\tau$ to a fixed finer set of roughly $4 N$ nodes, from which one computes (via the addition theorem) the coefficients of local expansions (i.e., Taylor expansions in the Laplace case, Fourier-Bessel expansions in the Helmholtz), around a set of expansion centers placed near (but not too near) $\partial \Omega$. It is these "surrogate" local expansions that are then evaluated at nearby desired target points $x$. This is reminiscent of the method of Schwab-Wendland [30] but with the major difference that expansion centers lie off of, rather than on, $\partial \Omega$. In section 4.2 we show how to combine the new scheme with the FMM to achieve

\footnotetext{
${ }^{1}$ In [21] this is called the "underlying" scheme, and in [18] "straight-up" quadrature.
} 
(a) $\log _{10}\left|u^{(N)}-u\right| \quad$ DLP, $\tau \equiv 1, \omega=0$
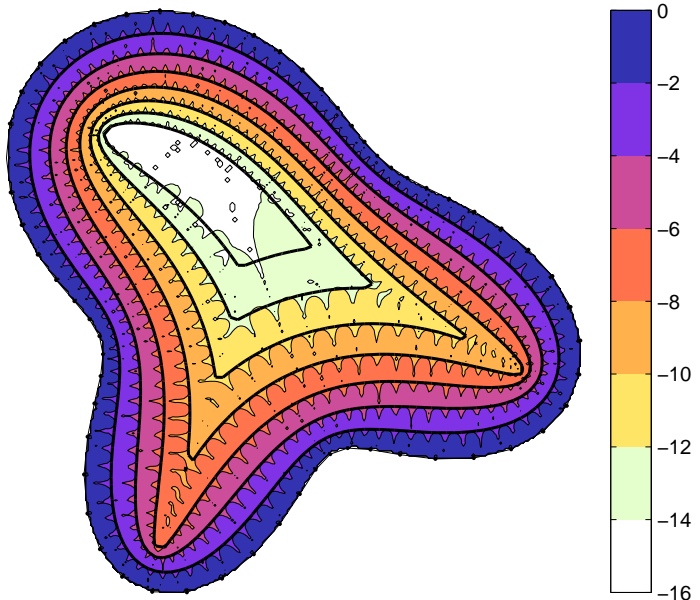

(c) $\log _{10}\left|u^{(N)}-u\right| \quad$ DLP, $\tau \equiv 1, \omega=0$

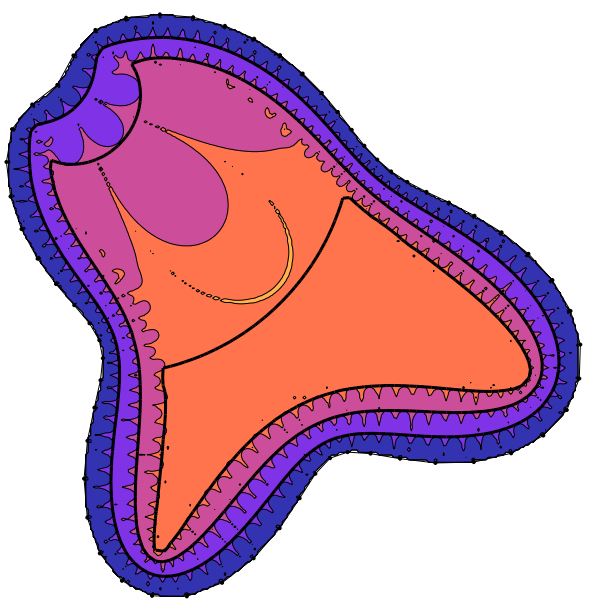

(b) $\log _{10}\left|u^{(N)}-u\right|$

DLP, BVP $u(x, y)=x y, \omega=0$

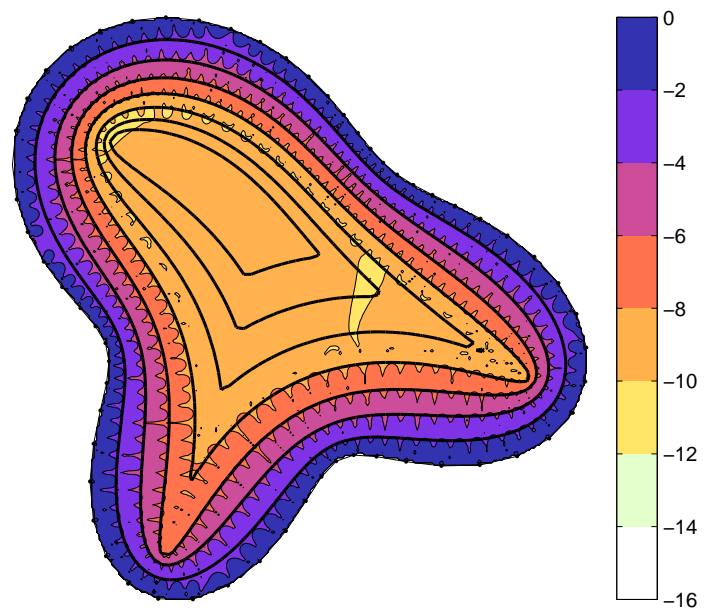

(d) $\log _{10}\left|u^{(N)}-u\right| \quad$ GRF, $u$ point src, $\omega=2$

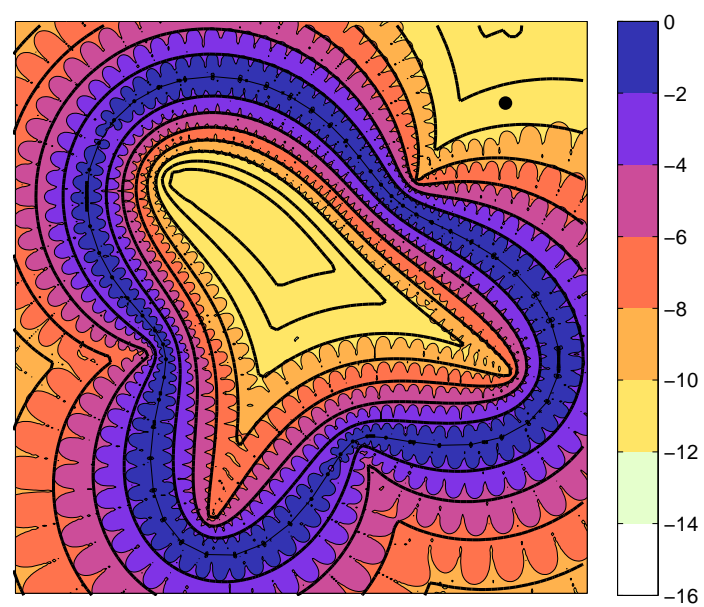

FIG. 1.1. Error in evaluation of layer potentials via the native quadrature on $N=60$ nodes, with contours every factor of $10^{2}$, and contours (thick black lines) predicted by Theorem 3. In (a), (b), and (d) the interior domain $\Omega$ has boundary given by the polar function $r(\theta)=1+0.3 \cos [3(\theta+0.3 \sin \theta)]$. (a) is a double-layer with $\tau \equiv 1$ and $\omega=0$. (b) $\tau$ is the Nyström solution for Dirichlet data corresponding to the potential $u(x, y)=x y$, with $\omega=0$. (c) same as (a) but $\partial \Omega$ has a small Gaussian "bump" on its northwest side. (d) Test of Green's representation formula (2.12), both inside and outside, for $\omega=2$ (roughly 0.8 wavelength across the diameter) with data due to an exterior point source at the dot shown.

an overall $O(N)$ complexity for the evaluation of $O(N)$ target points lying in the strip, and apply this to high-frequency scattering from a smooth but complicated obstacle 100 wavelengths across. Finally, we conclude and mention future directions in section 5.

2. Theory of Laplace layer potential evaluation error using the global trapezoid rule. Our goal in this section is to analyze rigorously the native (i.e., $N$-node) evaluation error for analytic single- and double-layer potentials for the Laplace equation $(\omega=0)$, on analytic curves. An example plot of such error varying over an interior domain is shown in Fig. 1.1(a). 


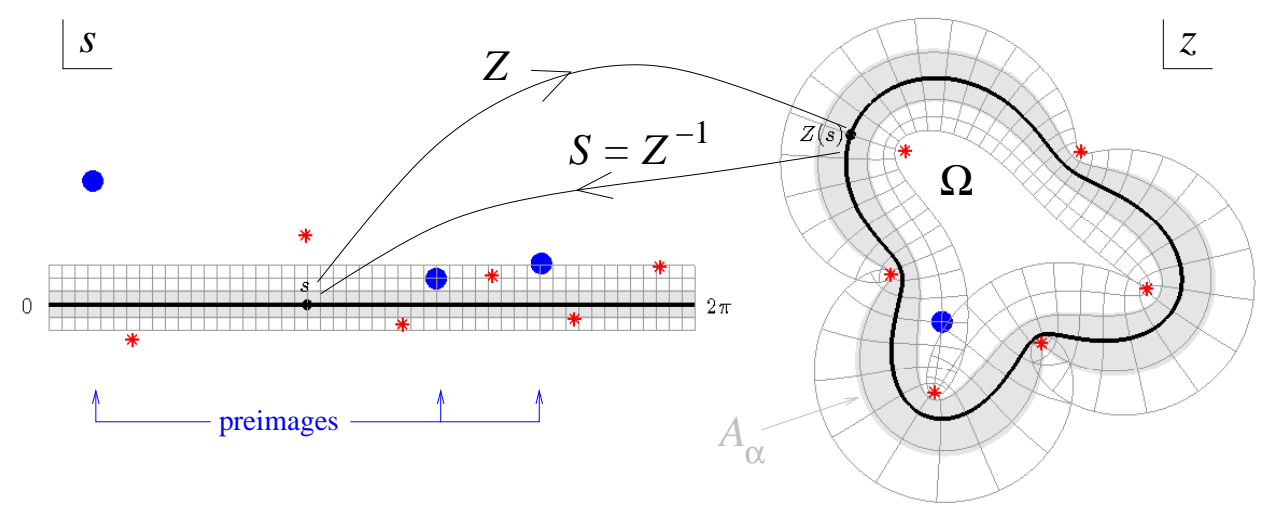

FIG. 2.1. Parametrization of the boundary of the interior domain $\Omega$ from Fig. 1.1, as a map from s-plane (shown left with a square grid) to z-plane (shown right). One point on the real $s$ axis and its image on the boundary are shown by small black dots. A single point in the interior and its three pre-images in the s-plane are shown by large blue dots. An annular strip $A_{\alpha}$ in which $Z$ is analytic and invertible, and its preimage, is shown in gray. The nearest six (branch-type) singularities of the Schwarz function of the domain, and their pre-images, are shown by $*$.

2.1. Geometric preliminaries. We identify $\mathbb{R}^{2}$ with $\mathbb{C}$, and let the simple analytic closed curve $\partial \Omega$ define either a bounded interior, or unbounded exterior, open domain $\Omega \subset \mathbb{C}$. We need $Z: \mathbb{R} \rightarrow \mathbb{C}$ as an analytic $2 \pi$-periodic counter-clockwise parametrization of $\partial \Omega$, i.e. $Z([0,2 \pi))=$ $\partial \Omega$. This means $Z(s)=z_{1}(s)+i z_{2}(s)$, with $z_{1}$ and $z_{2}$ real analytic and $2 \pi$-periodic, and that $Z$ may be continued as an analytic function in some neighborhood of the real axis. We assume that the speed function $\left|Z^{\prime}(s)\right|$ is positive for all real $s$. These conditions means that $Z$ is analytic and invertible in a strip $|\operatorname{Im} s|<\alpha$, for some $\alpha>0$. The image of this strip under $Z$ is an annular neighborhood of $\partial \Omega$ in which $Z^{-1}$ is also analytic. Fig. 2.1 shows such a strip and its image, both shaded in gray. Also shown are the singularities that control its width: at these points $Z^{\prime}=0$ so that $Z^{-1}$ ceases to be single-valued (in this example they are all square-root branchtype singularities, the generic case). These points are also singularities of the so-called Schwarz function of the domain; see [12, Ch. 5, 6, 8] and [22]. (Other types of Schwarz singularities are possible for analytic domains; e.g. see [3].)

For $\alpha \in \mathbb{R}$, we will use the notation $\Gamma_{\alpha}$ to mean a translation of $\partial \Omega$ by $\alpha$ in the imaginary parameter direction,

$$
\Gamma_{\alpha}:=Z(\{s=t+i \alpha: t \in \mathbb{R}\}) .
$$

In particular, $\Gamma_{0}=\partial \Omega$. Note that for all sufficiently small $\alpha, \Gamma_{\alpha}$ is a Jordan curve, but that for larger $|\alpha|$, it will generally start to self-intersect. This is illustrated by the images of the grid-lines in Fig. 2.1. For $\alpha>0$ we use $A_{\alpha}$ to denote the open annular strip region

$$
A_{\alpha}:=Z(\{s=t+i a: t, a \in \mathbb{R},|a|<\alpha\}) .
$$

Note that when neither $\Gamma_{-\alpha}$ nor $\Gamma_{\alpha}$ self-intersects, then $A_{\alpha}$ is the region lying between them.

2.2. Evaluation error in the double-layer case. Given a real-valued analytic density $\tau \in C(\partial \Omega)$, the Laplace double-layer potential (1.5) may be written $u=\operatorname{Re} v$, where $v$ is the 
complex contour integral ${ }^{2}$

$$
v(z)=\frac{-1}{2 \pi i} \int_{\partial \Omega} \frac{\tau(y)}{y-z} d y, \quad z \in \mathbb{C} \backslash \partial \Omega .
$$

Thus $v$ is analytic in $\Omega$, and also in $\mathbb{C} \backslash \bar{\Omega}$. Since the evaluation error of $u$ is bounded by that of $v$, we shall work with $v$ from now on. Let $\tilde{\tau}$ be the pullback of $\tau$ under $Z$, i.e. $\tilde{\tau}(s)=\tau(Z(s))$ for all $s \in \mathbb{R}$. Rewriting (2.1) in terms of the parameter gives,

$$
v(z)=\frac{-1}{2 \pi i} \int_{0}^{2 \pi} \frac{\tilde{\tau}(s)}{Z(s)-z} Z^{\prime}(s) d s, \quad z \in \mathbb{C} \backslash \partial \Omega .
$$

For quadrature of $(2.2)$ we now choose the global periodic trapezoid rule along the real $s$ axis, introducing nodes $2 \pi j / N, j=1, \ldots, N$, and equal weights $2 \pi / N$, thus

$$
v^{(N)}(z):=\frac{-1}{i N} \sum_{j=1}^{N} \frac{\tilde{\tau}(2 \pi j / N)}{Z(2 \pi j / N)-z} Z^{\prime}(2 \pi j / N) .
$$

The integrand in (2.2) is analytic, implying exponential convergence of (2.3) by the following classical theorem [11] (see e.g. [24, Thm. 12.6]).

TheOREM 1 (Davis). Let $f$ be $2 \pi$-periodic and analytic in the strip $|\operatorname{Im} s| \leq \alpha$ for some $\alpha>0$, and let $|f| \leq F$ in this strip. Then the quadrature error of the periodic trapezoid rule,

$$
E_{N}:=\frac{2 \pi}{N} \sum_{j=1}^{N} f(2 \pi j / N)-\int_{0}^{2 \pi} f(s) d s,
$$

obeys the bound

$$
\left|E_{N}\right| \leq \frac{4 \pi F}{e^{\alpha N}-1}
$$

However, to achieve (rather than merely approach) the correct convergence rate, we will need the following generalization (similar to that of Hunter [20]):

Lemma 2. Let $f$ be $2 \pi$-periodic and meromorphic in the strip $|\operatorname{Im} s| \leq \alpha$ for some $\alpha>0$, with only one simple pole in this strip, at $s_{0}$, with $\operatorname{Im} s_{0} \neq 0$. Let $f$ have residue $r_{0}$ at this pole, and let $|f| \leq F$ on the edges of the strip, i.e. for all $s$ with $|\operatorname{Im} s|=\alpha$. Then the quadrature error (2.4) obeys the bound

$$
\left|E_{N}\right| \leq \frac{2 \pi r_{0}}{e^{\left|\operatorname{Im} s_{0}\right| N}-1}+\frac{4 \pi F}{e^{\alpha N}-1} .
$$

Note that the first term dominates as $N$ grows, and that $r_{0}=0$ recovers the Davis theorem.

Proof. Let $\Gamma_{1}$ and $\Gamma_{2}$ be the upper and lower strip boundaries respectively, both traversed with increasing real part. For the sum in (2.4) we apply the residue theorem to $\cot \frac{N s}{2} f(s)$ in the strip, noticing that the vertical sides cancel due to periodicity. For the integral in (2.4) we apply the residue theorem to $f(s)$ in each of the upper and lower semi-strips, take their average. Combining these, (2.4) can be rewritten

$$
E_{N}=\int_{\Gamma_{1}}\left(\frac{i}{2} \cot \frac{N s}{2}-\frac{1}{2}\right) f(s) d s-\int_{\Gamma_{2}}\left(\frac{i}{2} \cot \frac{N s}{2}+\frac{1}{2}\right) f(s) d s+2 \pi i r_{0}\left(\frac{i}{2} \cot \frac{N s_{0}}{2} \mp \frac{1}{2}\right),
$$

\footnotetext{
${ }^{2}$ Note that this Cauchy integral is not an example of Cauchy's theorem, because $\tau$ is not the boundary value of $v$. Rather, $\tau$ is purely real-valued.
} 
with the choice of sign in the last term corresponding to the cases where $\operatorname{Im} s_{0}$ has sign \pm . The first bracketed term is bounded in size by $\left(e^{\alpha N}-1\right)^{-1}$ since $\operatorname{Im} s=\alpha$. The same is true for the second bracketed term since $\operatorname{Im} s=-\alpha$. The third bracketed term is bounded by $\left(e^{\left|\operatorname{Im} s_{0}\right| N}-1\right)^{-1}$. Combining these estimates and the bound on $f$ on the strip boundary completes the proof. $\square$

Now, since $\tau$ is real analytic on $\partial \Omega$, it may be continued as a bounded holomorphic function in the closure of some annular strip $A_{\alpha}$. Let us choose $\alpha>0$ so that $Z$ is also analytic and invertible in the closure of $A_{\alpha}$ as discussed in section 2.1. This is sufficient for the pullback $\tilde{\tau}$ to be bounded and holomorphic in the closed $s$-plane strip of half-width $\alpha$. Consider a target evaluation point $z \in A_{\alpha}$, which then has a unique preimage $s=Z^{-1}(z)$ with $|\operatorname{Im} s|<\alpha$. Recalling the native evaluation (2.3), define the error function

$$
\epsilon_{N}:=\operatorname{Re}\left(v^{(N)}-v\right) .
$$

Applying Lemma 2 in the strip $|\operatorname{Im} s|<\alpha$, noticing that the residue of the integrand in (2.2) is just $\tilde{\tau}(s)$, and bounding the dominant first term in (2.6) by a simple exponential, we have shown:

THEOREM 3. Let $A_{\alpha}, \alpha>0$, be an annular strip in the closure of which $\tau$ is holomorphic and bounded, and $Z^{-1}$ is holomorphic. Then at each target $z \in A_{\alpha} \backslash \partial \Omega$ we have exponential convergence of the error of the Laplace double-layer potential evaluated with the $N$-point trapezoid rule. That is, there exists constants $C$ and $N_{0}$ such that

$$
\left|\epsilon_{N}(z)\right| \leq C e^{-|\operatorname{Im} s| N} \quad \text { for all } N \geq N_{0}
$$

where $Z(s)=z$. The constant $C$ may be chosen to be any number greater than $|\tilde{\tau}(s)|=|\tau(z)|$. To summarize: convergence is exponential with rate given by the imaginary part of the preimage of the target point under the complexification of the boundary parametrization.

REMARK 4. It is possible to choose constants $C$ and $M$ for which (2.8) holds uniformly in any compact subset of $A_{\alpha} \backslash \partial \Omega$, but this is impossible over the entire set $A_{\alpha} \backslash \partial \Omega$ because the $N$ at which exponential convergence sets in diverges as $1 /|\operatorname{Im} s|$ as one approaches $\partial \Omega$. Intuitively, this failure occurs because, no matter how large $N$ is, individual quadrature points are always "visible" close enough to the boundary. However, the constant $C$ may be chosen uniformly on the entire set to be any number greater than $\sup _{z \in A_{\alpha}}|\tau(z)|$.

In Fig. 1.1 (a) we plot contours of constant $\epsilon_{N}(z)$ as the target point $z$ is varied over a nonsymmetric interior domain $\Omega$ with analytic boundary, for fixed $N$, and the simplest case $\tau \equiv 1$ which generates the potential $u \equiv-1$ in $\Omega$. We overlay (as darker curves) predicted contours using Theorem 3. In the annular strip $A_{\alpha}$ (shown in gray in Fig. 2.1) the predicted contour for an error level $\epsilon$ is the curve $\Gamma_{-} \log (\epsilon / C) / N$, where the lower bound $C=1$ was used. The match between the light and dark contours is almost perfect (apart from periodic "scalloping" due to oscillation in the error at the node frequency).

REMARK 5. Note that it would be possible to use the properties of the cot function to improve Theorem 3 to include a lower bound on $\left|v^{(N)}(z)-v(z)\right|$ asymptotically approaching the upper bound. We have not pursued this, since after taking the real part the error has no lower bound; rather, it oscillates in sign at the node frequency as shown by the "fingers" in Fig. 1.1 (a).

What happens further into the domain $\Omega$, i.e. for $\alpha$ values larger than that for which $Z$ is invertible? Here, since $Z^{-1}$ starts to become multi-valued, there are multiple preimages which lie within a given strip $|\operatorname{Im} s| \leq \alpha$ (e.g. see large dots in Fig. 2.1). To analyze this would require a variant of Lemma 2 with multiple poles. We prefer an intuitive explanation. Let us assume that $\tilde{\tau}$ remains holomorphic throughout such a wider strip. Then it is clear that the $s$-plane pole closest to the real axis will dominate the error for sufficiently large $N$ because it creates the slowest exponential decay rate. Hence, to generate each predicted contour in Fig. 1.1 we use the set of $z$-plane points which have their closest preimage a distance $\alpha=-\log (\epsilon / C) / N$ from the real axis. For each $\alpha$, this curve is simply the boundary of $A_{\alpha}$, i.e. the self-intersecting curve $\Gamma_{\alpha}$ 
with all its "loops trimmed off." We see in Fig. 1.1(a) that, throughout the interior of $\Omega$, this leads to excellent prediction of the error down to at least 14 digits of accuracy. Even features such as the cusps which occur beyond the two closest interior Schwarz singularities (at roughly 4 o'clock and 7 o'clock) are as predicted.

Eventually, for a target point deep inside the domain, all of its preimages may be further from the real axis than the widest strip in which $\tilde{\tau}$ is holomorphic. In this case, one is able to apply only the Davis theorem, and the width of the strip in which $\tilde{\tau}$ is holomorphic will now control the error (this case is never reached in Fig. 1.1(a), although it will be in (d)).

We now perform some instructive variants on this numerical experiment. In Fig. 1.1(b) we use the same domain and $N$ as in (a), but instead of using a given $\tau$, we solve for $\tau$ via (1.4) with the $N$-point Nyström method, given (entire) Dirichlet data $u(x, y)=x y$. This is a typical BVP setting, albeit a simple one. We see that errors are similar to (a) with the major difference that errors bottom out at around $10^{-9}$ : this is because $\tau$ itself only has this accuracy for the $N=60$ nodes used $(N \geq 130$ recovers full machine precision in $\tau)$.

In (c) we repeat (a) except using a boundary shape $\partial \Omega$ distorted by a localized Gaussian "bump" at around 11 o'clock. Errors are now never smaller than $10^{-8}$ : note that since $\tau \equiv 1$ this cannot be due to inaccuracy, nor to lack of sufficient analyticity, in $\tau$. Rather, the mechanism is the rapid growth in distance from $\partial \Omega$ of the contours $\Gamma_{\alpha}$ in this region, as $\alpha$ increases. This is verified by the quite good agreement with predicted contours. We observe that such growth is typical in a region with rapidly-changing curvature, which explains the well-known empirical rule that, for high accuracy, $N$ must be chosen large enough to resolve such spatial features.

To remind the reader that Theorem 3 predicts errors just as well in the exterior as in the interior, we suggest a glance at Fig. 1.1(d), to be discussed more later. We conclude with a remark about the universality of the "safe" distance from the boundary for accurate evaluation.

REMARK 6 ( $5 h$ rule). In practical settings, if the evaluation point is a distance $5 h$ or more from the boundary, where $h$ is the local spacing between the nodes $Z(2 \pi j / N)$, then around 14 digits of accuracy in $u^{(N)}$ is typical. This is because, when the local distortion induced by the conformal map $Z$ is small, the preimage is then a distance roughly $5 \cdot 2 \pi / N$ from the real axis, giving the term $e^{-5 \cdot 2 \pi} \approx 2 \times 10^{-14}$ in (2.8). This relies on two assumptions: i) $\tau$ is analytic and bounded in a strip of sufficient width, and ii) the local distortion is small on a spatial scale of a few times $h$. Why should these hold in practice? The answer is that they are preconditions for the Nyström method to produce a highly accurate solution density $\tau$ in the first place.

Finally, we note that if the periodic trapezoid rule were to be replaced by a panel-based quadrature with Chebyshev node density, such as Gauss-Legendre, a similar analysis to the above would show that the contours of error level are the images under $Z$ of the Bernstein ellipses $[13,33]$ for the panel intervals.

2.3. Single-layer case. We now present a similar analysis for single-layer potential evaluation, including a numerical verification. Recalling the fundamental solution (1.3), the single-layer potential is

$$
u(x)=\int_{\partial \Omega} \Phi(x, y) \sigma(y) d s_{y}, \quad x \in \mathbb{C} \backslash \partial \Omega
$$

In the Laplace case $(\omega=0)$, a real-valued analytic density $\sigma \in C(\partial \Omega)$ generates a potential $u=\operatorname{Re} v$, where, analogously to (2.1) and (2.2),

$$
v(z)=\frac{1}{2 \pi} \int_{\partial \Omega}\left(\log \frac{1}{y-z}\right) \sigma(y)|d y|=\frac{1}{2 \pi} \int_{0}^{2 \pi}\left(\log \frac{1}{Z(s)-z}\right) \tilde{\sigma}(s)\left|Z^{\prime}(s)\right| d s, \quad z \in \mathbb{C} \backslash \partial \Omega,
$$


and $\tilde{\sigma}(s):=\sigma(Z(s))$ is the pullback. Note that now the magnitude of $d y$ rather than its complex value is taken. As before, we will assume that the analytic continuation of $\tilde{\sigma}$ is analytic in some strip $|\operatorname{Im} s| \leq \alpha$ in which $Z$ is analytic and invertible. The following is a variation on Lemma 2 .

LEMMA 7. Let $f$ be $2 \pi$-periodic and analytic everywhere in the strip $|\operatorname{Im} s| \leq \alpha$ apart from on a branch cut $\Gamma_{C}$ which starts from the point $s_{0}$, with $\operatorname{Im} s_{0} \neq 0$, then proceeds to the nearest edge of the strip while avoiding the region $|\operatorname{Im} s| \leq\left|\operatorname{Im} s_{0}\right|$. Let $|f| \leq F$ on the edges of the strip. Then the quadrature error (2.4) obeys the bound

$$
\left|E_{N}\right| \leq \frac{1}{e^{\left|\operatorname{Im} s_{0}\right| N}-1} \int_{\Gamma_{C}}\left|f^{+}(s)-f^{-}(s)\right| d s+\frac{4 \pi F}{e^{\alpha N}-1},
$$

where $f^{+}$and $f^{-}$are the limiting values on either side of the branch cut.

Proof. As in the proof of Lemma 2, we use the residue and Cauchy theorems to write $E_{N}$ as

$\int_{\Gamma_{1}}\left(\frac{i}{2} \cot \frac{N s}{2}-\frac{1}{2}\right) f(s) d s-\int_{\Gamma_{2}}\left(\frac{i}{2} \cot \frac{N s}{2}+\frac{1}{2}\right) f(s) d s+\int_{\Gamma_{C}}\left(\frac{i}{2} \cot \frac{N s}{2} \mp \frac{1}{2}\right)\left[f^{+}(s)-f^{-}(s)\right] d s$,

taking care to include the traversal of both sides of $\Gamma_{C}$ in the relevant contours. As before, the first two integrals may be bounded to give the second term in (2.11). The last integral may be bounded by the first term in (2.11).

We now apply this to the native evaluation error for the single-layer potential (2.10), and find exponential convergence with the same rate as for the double-layer.

THEOREM 8. Let $A_{\alpha}, \alpha>0$, be a annular strip in the closure of which $\sigma$ is holomorphic and bounded, and $Z^{-1}$ is holomorphic. Let $\epsilon_{N}$ be the evaluation error function of the Laplace single-layer potential $u=\operatorname{Re} v$ given by (2.10) with the $N$-point periodic trapezoid rule. Then at each target $z \in A_{\alpha} \backslash \partial \Omega$, there exists constants $C$ and $N_{0}$ such that

$$
\left|\epsilon_{N}(z)\right| \leq C e^{-|\operatorname{Im} s| N} \quad \text { for all } N \geq N_{0}
$$

where $Z(s)=z$. The constant $C$ may be chosen to be any number larger than an upper bound on $|\tilde{\sigma}|$ on $\Gamma_{C}$ times the geometric mean of the length of the curves $Z\left(\Gamma_{C}\right)$ and $Z\left(\overline{\Gamma_{C}}\right)$, where $\Gamma_{C}$ is any cut as in Lemma 7.

Proof. We use the notation $\bar{s}$ to indicate the complex conjugate of $s$. We note that the analytic continuation of $\left|Z^{\prime}(s)\right|$ off the real axis is $\left(Z^{\prime}(s) \overline{Z^{\prime}(\bar{s})}\right)^{1 / 2}$, which is analytic and bounded in the strip. Thus we set $f(s)=(1 / 2 \pi)[\log 1 /(Z(s)-z)] \tilde{\sigma}(s)\left(Z^{\prime}(s) \overline{Z^{\prime}(\bar{s})}\right)^{1 / 2}$ in Lemma 7 , and note that the jump in the logarithm everywhere on the branch cut is $2 \pi i$. Then

$$
\int_{\Gamma_{C}}\left|f^{+}(s)-f^{-}(s)\right| d s \leq \sup _{s \in \Gamma_{C}}|\tilde{\sigma}(s)| \cdot \int_{\Gamma_{C}}\left|Z^{\prime}(s)\right|^{1 / 2}\left|Z^{\prime}(\bar{s})\right|^{1 / 2} d s,
$$

to which we apply the Cauchy-Schwarz inequality. As in Theorem 3, we bound the first exponential term in (2.11); the smaller second term can be absorbed into the constant.

We now test the convergence for a combination of single- and double-layer densities, by checking the accuracy of the following Green's representation formula (GRF). For $u$ a solution to the PDE (1.1) in a bounded domain $\Omega \subset \mathbb{R}^{2}$,

$$
\int_{\partial \Omega} \Phi(x, y) \frac{\partial u}{\partial n}(y) d s_{y}-\int_{\partial \Omega} \frac{\partial \Phi(x, y)}{\partial n(y)} u(y) d s_{y}= \begin{cases}u(x), & x \in \Omega \\ 0, & x \in \mathbb{R}^{2} \backslash \bar{\Omega} .\end{cases}
$$

Errors for the native evaluation of this in both interior and exterior cases are shown together in Fig. 1.1(d): it is clear that errors grow exponentially as $x$ approaches $\partial \Omega$, from either inside 
(a) $\log _{10}\left|u^{(N)}-u\right| /\|u\|_{\infty}$, DLP, $u(x, y)=e^{y} \cos x$

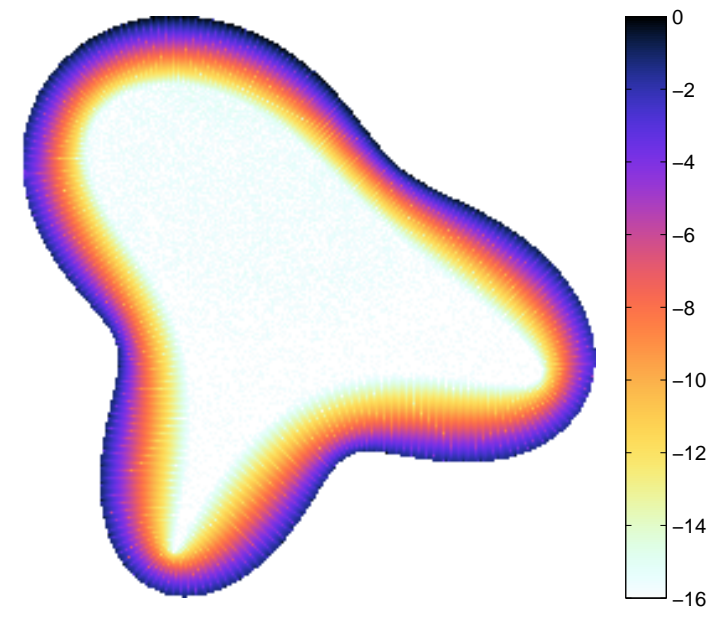

(b) $\log _{10}|\hat{u}-u| /\|u\|_{\infty}$, DLP, $u(x, y)=e^{y} \cos x$

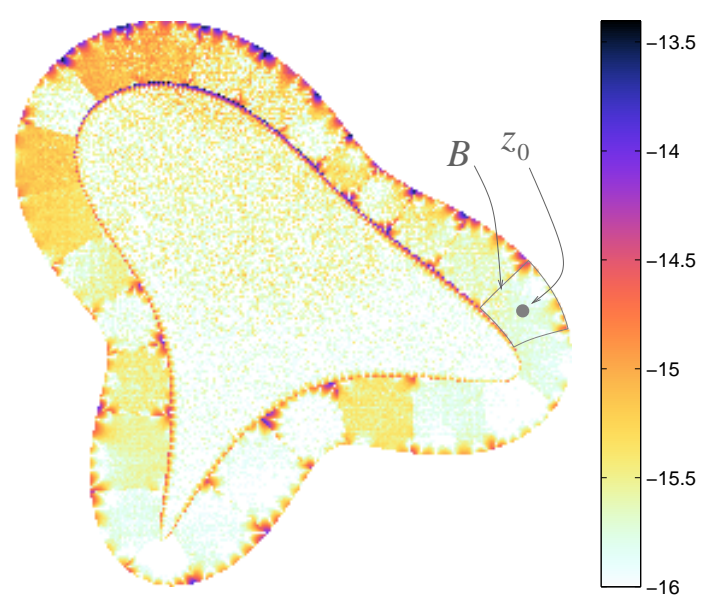

FIG. 2.2. Error (relative to largest value) of evaluation of solution of Laplace BVP with Dirichlet data $u(x, y)=e^{y} \cos x$, with $N=130$, using the native quadrature (a), and the proposed scheme (b) with $N_{B}=26$, $p=10$ and $M=4 N$. Note the change in color scale: relative $L^{\infty}$ error is $4 \times 10^{-14}$, and relative $L^{2}$ error $4 \times 10^{-15}$. One box $B$ and its center $z_{0}$ are labeled; many other boxes and the the annular half-strip $\Omega_{\text {bad }}=A_{\text {bad }} \cap \Omega$ are visible due to jumps in error.

or outside. In fact, this figure shows the case for the low-frequency Helmholtz equation with $\omega=2$; the Laplace plot is almost identical. This verifies the intuition that the convergence for low-frequency Helmholtz is very similar to Laplace. (See section 4 for the formulae for the Helmholtz case.)

Why do the errors stop decreasing with distance once $10^{-12}$ is reached in Fig. 1.1(d)? This cannot be due to inaccurate boundary data, since the data is exact to machine precision. Rather, the answer lies in the fact that the boundary data derives from a point source (shown by a large dot) outside, but not too far from, $\Omega$. This means that the densities cannot be analytic in a larger annular strip, limiting the maximum convergence rate in $N$ to the value at this singularity location. This illustrates the effect of densities which are not entire functions.

3. Close evaluation of Laplace layer potentials by surrogate local expansions. In this section we present and analyze the main new scheme. We have seen that the native evaluation error is exponentially small far from $\partial \Omega$, but intolerably large close to $\partial \Omega$. Thus we define the "bad annular strip" $A_{\text {bad }}:=A_{\alpha_{\text {bad }}}$ where, following Remark 6 , we will choose $\alpha_{\text {bad }}=10 \pi / N$, that is, a distance around $5 h$ either side of $\partial \Omega$, giving around 14 digit expected accuracy throughout $\mathbb{R}^{2} \backslash A_{\text {bad }}$. (Obviously $\alpha_{\text {bad }}$ could be decreased if less accuracy is desired.) We now describe the new method for layer potential evaluation in this bad annular strip, focusing on the case of $\Omega_{\text {bad }}:=A_{\text {bad }} \cap \Omega$, for $\Omega$ an interior domain with $\partial \Omega$ traversed in the counter-clockwise sense. Thus, target preimages will have positive imaginary part. (The exterior case is analogous.)

Let us fix $N$ and assume that a double-layer potential $\tau$ has been approximately computed by the Nyström method, so is represented by $\left\{\tau_{j}\right\}_{j=1}^{N}$, its values at the nodes. A precondition for Nyström accuracy is that $\tau$ is well-approximated by its Nyström interpolant through these nodes. Recalling that $u=\operatorname{Re} v$, we wish to evaluate $v$ via $(2.1)$ in $\Omega_{\text {bad }}$. We cover $\Omega_{\text {bad }}$ by non-intersecting "boxes" $B_{b}, b=1, \ldots, N_{B}$. The $b$ th box $B_{b}$ is the image under $Z$ of the $s$-plane rectangle $2 \pi(b-1 / 2) / N_{B} \leq \operatorname{Re} s<2 \pi(b+1 / 2) / N_{B}, 0 \leq \operatorname{Im} s \leq \alpha_{\text {bad }}$. By choosing $N_{B}=\lceil N / 5\rceil$ the boxes become very close to being square. Fig. 2.2(b) shows the annular half-strip and one box. 
Let $B=B_{b}$ be the $b$ th box. We choose an expansion center

$$
z_{0}:=Z\left(2 \pi b / N_{B}+i \alpha_{0}\right)
$$

where the choice of imaginary distance $\alpha_{0}=\alpha_{\text {bad }} / 2$ places $z_{0}$ roughly central to $B$, and roughly $2.5 \mathrm{~h}$ from the boundary. We represent $v$ by a Taylor series

$$
v(z)=\sum_{m=0}^{\infty} c_{m}\left(z-z_{0}\right)^{m}
$$

which converges uniformly in $B$ if $v$ is analytic in some disc centered at $z_{0}$ with radius greater than $R$, where $R$ is the maximum radius of the box,

$$
R:=\sup _{z \in B}\left|z-z_{0}\right| .
$$

Each coefficient $c_{m}$ can be computed, using the Cauchy formula for derivatives, as

$$
c_{m}=\frac{-1}{2 \pi i} \int_{\partial \Omega} \frac{\tau(y)}{\left(y-z_{0}\right)^{m+1}} d y=\frac{-1}{2 \pi i} \int_{0}^{2 \pi} \frac{\tilde{\tau}(s)}{\left(Z(s)-z_{0}\right)^{m+1}} Z^{\prime}(s) d s, \quad m=0,1, \ldots
$$

To approximate the latter integral we use the periodic trapezoid rule with $M$ new nodes, $s_{j}=$ $2 \pi j / M, j=1, \ldots, M$, which we call "fine" nodes, that is,

$$
c_{m} \approx \hat{c}_{m}:=\frac{-1}{i M} \sum_{j=1}^{M} \frac{\tilde{\tau}\left(s_{j}\right)}{\left(Z\left(s_{j}\right)-z_{0}\right)^{m+1}} Z^{\prime}\left(s_{j}\right) .
$$

Since $z_{0}$ is in the bad annular strip, clearly we need $M>N$ to evaluate even the $m=0$ coefficient accurately: one would expect, since $\alpha_{0}=\alpha_{\text {bad }} / 2$, that $M=2 N$ would be sufficient. However, for large $m$ the term $1 /\left(Z(s)-z_{0}\right)^{m+1}$ is now oscillatory, so an even larger $M$ will be needed. Note that $\tilde{\tau}\left(s_{j}\right)$ must be found by interpolation from its values at the $N$ Nyström nodes. Our surrogate potential in the box $B$ is then simply the truncated series with the above approximated coefficients, and $p$ terms, i.e.

$$
v(z) \approx \hat{v}(z)=\sum_{m=0}^{p-1} \hat{c}_{m}\left(z-z_{0}\right)^{m}, \quad z \text { in box } B
$$

Finally the real part must be taken, thus in each of the boxes we set $\hat{u}=\operatorname{Re} \hat{v}$. We claim that, with the parameters $p$ and $M$ well chosen, then $\hat{u} \approx u$ uniformly to high accuracy throughout $\Omega_{\text {bad }}$. (In $\Omega \backslash \Omega_{\text {bad }}$ we revert to $\hat{u}=u^{(N)}$, the native evaluation scheme.)

This is illustrated in Fig. 2.2: we first solve (1.4) for $\tau$, given entire boundary data, using $N=130$, which is around the minimum $N$ required to achieve full accuracy in $\tau$. We then try the new evaluation scheme, using a standard trigonometric polynomial interpolant (e.g. see [24, Sec. 11.3], implemented easily via the FFT) to approximate $\tau$ at the fine nodes, which gives a $\hat{u}$ with uniform relative accuracy of 13.5 digits (14 digits in the $L^{2}(\Omega)$ norm). Note that, since the boundary data is entire, this is a well-behaved example; we consider more challenging cases after the following analysis.

3.1. A convergence theorem for the Laplace double-layer potential. Because the boxes touch $\partial \Omega$, uniform convergence of the Taylor series of $v$ in any box generally demands that $v$ have an analytic continuation some distance outside $\Omega$, which might seem far-fetched. But it turns out that the region of analyticity of $v$ is at least as large as that of $\tau$. 
Proposition 9. Let $\tau$ be analytic in some closed annular neighborhood $A$ of $\partial \Omega$. Then $v$ given by (2.2) is analytic in $\Omega$ and moreover continues as an analytic function throughout $A \backslash \Omega$.

Proof. Let $\Gamma$ be the exterior boundary of $A$. Consider the identity arising from (2.1),

$$
\frac{-1}{2 \pi i} \int_{\Gamma} \frac{\tau(y)}{y-z} d y=v(z)+\frac{-1}{2 \pi i} \int_{\Gamma-\partial \Omega} \frac{\tau(y)}{y-z} d y,
$$

where $-\partial \Omega$ means $\partial \Omega$ traversed in the opposite direction. Both sides equal $v(z)$ for $z \in \Omega$ since the second term on the right is zero by Cauchy's theorem. However, as a function of $z$, the left side is analytic throughout $A \cup \Omega$, and thus provides the desired analytic continuation of $v$.

To state a convergence result, some control is needed of the distortion induced by the conformal map $Z$. Let $A_{\alpha}$ be an annular strip in which $Z^{-1}$ exists. Recall that box $B$ has a center $z_{0}$ (which we assume is in $A_{\alpha}$ ) with $\alpha_{0}$ the imaginary part of its preimage, and radius $R$. Given this, we define a geometric distortion quantity,

$$
\gamma=\gamma_{z_{0}, R}:=\sup _{0<a<\alpha_{0}} \frac{R}{d\left(\Gamma_{a}, z_{0}\right)} \frac{\alpha_{0}-a}{\alpha_{0}},
$$

where $d(X, y)$ means the minimum Euclidean distance from the point $y$ to points in the set $X$. It is clear that, as $a>0$ approaches $\alpha_{0}$ from below, the curve $\Gamma_{a}$ first touches $z_{0}$ at $a=\alpha_{0}$; an interpretation of $\gamma^{-1}$ is a scaled lower bound on the ratio between its distance and $\alpha_{0}-a$ (we note that in peculiar geometries it may be that the nearest part of $\Gamma_{a}$ is not within the box $B$.) For an undistorted square box with $z_{0}$ at its center, $\gamma=\sqrt{2}$; in practical settings $\gamma$ is around 1.5 to 2. For the geometry in Fig. 2.2(b), the median $\gamma$ is 1.7 and the maximum 2.4 (occurring at around 7 o'clock, where the closest interior Schwarz singularity lies). ${ }^{3}$

Our result concerns convergence of the surrogate scheme simultaneously in the expansion order $p$ and number of fine nodes $M$.

THEOREM 10. Let $B$ be a box with radius $R$. Let the center $z_{0}$ have $\operatorname{Im} z_{0}=\alpha_{0}$, with $A_{\alpha_{0}}$ an annular strip in which $Z^{-1}$ is holomorphic, and in which the density $\tau$ is holomorphic and bounded. Let the double-layer potential $v$ given by (2.1) be analytic in an open neighborhood of some closed disc of radius $\rho>R$ about $z_{0}$. Let $\hat{v}$ be given by the surrogate scheme (3.4) with $\hat{c}_{m}$ given by (3.3), using the exact density at the fine nodes $\tilde{\tau}\left(s_{j}\right)=\tau\left(Z\left(s_{j}\right)\right), j=1, \ldots, M$. Then the error function

$$
\hat{\epsilon}=\operatorname{Re}(\hat{v}-v)
$$

has the uniform bound

$$
\hat{\epsilon}_{B}:=\sup _{z \in B}|\hat{\epsilon}(z)| \leq C\left(\frac{R}{\rho}\right)^{p}+C\left(e \gamma \alpha_{0} M\right)^{p} p^{1-p} e^{-\alpha_{0} M} \quad \text { for all } M \geq 1 / \alpha_{0}, 1 \leq p \leq M / 2,
$$

where $C$ indicates constants that depend on $\partial \Omega, Z, \tau, B$ and $z_{0}$, but not on $p$ nor $M$.

The restriction to $M \geq 1 / \alpha_{0}$ always holds in practice because $N$ is already many times larger than $1 / \alpha_{0}$, whilst $M>N$. The restriction on $p$ also holds in practice, since usually $M>10^{2}$. The first term in (3.6) is simply the truncation error of the Taylor series for $v$ in the box, so is independent of $M$. Note that the required analyticity of $v$ is already given by Prop. 9 and the analyticity of $\tau$, with $\rho \approx \sqrt{2} R$, unless the local distortion is very large.

The $M$ dependence of the second term may be written $e^{-\alpha_{0} M+p \log M}$, showing that (at fixed $p$ ) this term is asymptotically exponentially convergent in $M$ with rate $\alpha_{0}$. Thus the whole

\footnotetext{
${ }^{3}$ These $\gamma$ values are accurately estimated using 20 values of $\alpha$ with $10^{3}$ points on each curve $\Gamma_{\alpha}$.
} 
scheme is also exponentially convergent: given arbitrary $\epsilon>0$, by fixing $p$ such that the first term is smaller than $\epsilon / 2$, one may then find an $M$ such that the second term is also smaller than $\epsilon / 2$, and have $\hat{\epsilon}_{B} \leq \epsilon$. There is a subtlety. Fixing $M$ while increasing $p$ is a bad idea: in practice it leads to exponential divergence, because the factor $e \gamma \alpha_{0} M$ is usually large (around 300 , independent of the problem size), and hence the second term grows exponentially in $p$ for typical $p$ (less than 30 ).

Proof. We will use $C$ to indicate (different) constants that have only the dependence stated in the theorem. Comparing $\hat{v}$ to the exact Taylor series for $v$, and using $\left|z-z_{0}\right| \leq R$,

$$
\hat{\epsilon}_{B} \leq \sup _{z \in B}\left|\sum_{m=0}^{p-1} \hat{c}_{m}\left(z-z_{0}\right)^{m}-v(z)\right| \leq \sum_{m \geq p}\left|c_{m}\right| R^{m}+\sum_{m=0}^{p-1}\left|\hat{c}_{m}-c_{m}\right| R^{m} .
$$

For the first term we use $\left|c_{m}\right| \leq C / \rho_{m}$, which follows from the Cauchy integral formula in the disc of radius $\rho$ (e.g. [31, Cor. 4.3]), and bound the geometric sum. For the second term we apply Theorem 1 to the $s$-integral (3.2) in a strip of width $\alpha$, so that, for each $\alpha \in\left(0, \alpha_{0}\right)$,

$$
\left|\hat{c}_{m}-c_{m}\right| \leq\left(\sup _{\operatorname{Im} s= \pm \alpha}\left|\tilde{\tau}(s) Z^{\prime}(s)\right|\right) \frac{2}{d\left(\Gamma_{\alpha}, z_{0}\right)^{m+1}} \frac{1}{e^{\alpha M}-1} \leq \frac{C}{d\left(\Gamma_{\alpha}, z_{0}\right)^{m+1}} \frac{1}{e^{\alpha M}-1}
$$

where the second estimate follows from the maximum modulus principle and boundedness of $Z^{\prime}$ and $\tilde{\tau}$ in the $\alpha_{0}$-strip. Inserting the above two estimates into (3.7), then bounding each term in the sum by the last term since $R>d\left(\Gamma_{\alpha}, z_{0}\right)$, gives

$$
\begin{aligned}
\hat{\epsilon}_{B} & \leq \frac{C}{1-R / \rho}\left(\frac{R}{\rho}\right)^{p}+\sum_{m=0}^{p-1} R^{m} \frac{C}{d\left(\Gamma_{\alpha}, z_{0}\right)^{m+1}} \frac{1}{e^{\alpha M}-1} \leq C\left(\frac{R}{\rho}\right)^{p}+C p\left(\frac{R}{d\left(\Gamma_{\alpha}, z_{0}\right)}\right)^{p} \frac{1}{e^{\alpha M}-1} \\
& \leq C\left(\frac{R}{\rho}\right)^{p}+C p\left(\frac{\gamma \alpha_{0}}{\alpha_{0}-\alpha}\right)^{p} e^{-\alpha M \quad \text { for all } M>1 / 2 \alpha,}
\end{aligned}
$$

where in the last step we used (3.5) and simplified the exponential bound. This estimate holds for each $\alpha \in\left(0, \alpha_{0}\right)$; however, if $\alpha$ is chosen too small, the last exponential will decay very slowly. Conversely, if $\alpha$ approaches $\alpha_{0}$ then the distance $d\left(\Gamma_{\alpha}, z_{0}\right)$ vanishes and its $p$ th negative power blows up rapidly. For each $p$ and $M$, the optimal value $\hat{\alpha}$ which minimizes this second term is found by setting $\partial / \partial \alpha$ of the logarithm of this term to zero, and solving, giving

$$
\hat{\alpha}=\alpha_{0}-\frac{p}{M}
$$

which by the condition on $p$ is never smaller than $\alpha_{0} / 2$. Substituting $\alpha=\hat{\alpha}$ in (3.9) gives (3.6).

To interpret the theorem we need to relate it to the original $N$ Nyström nodes (notice that $N$ does not appear in the theorem). We introduce two dimensionless parameters. Let

$$
\delta:=\frac{\alpha_{0} N}{2 \pi}
$$

be the center $z_{0}$ distance from the boundary in units of local node spacing $h$; recall that we set $\delta=2.5$ in the above. Let

$$
\beta:=\frac{M}{N}
$$

be the "upsampling ratio", the ratio of the number of fine nodes to original Nyström nodes. 
(a) $\log _{10}\|\hat{u}-u\|_{\infty} /\|u\|_{\infty}, \quad N=130$ $u(x, y)=e^{y} \cos x$
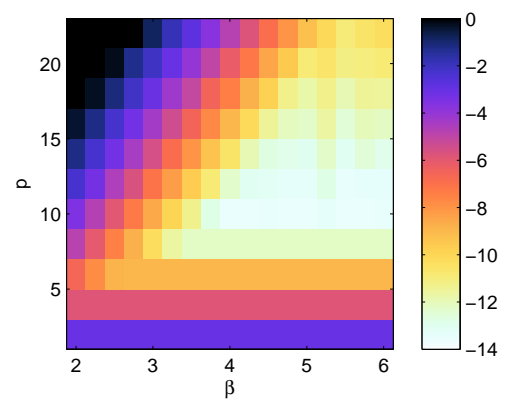

(b) $\log _{10}\|\hat{u}-u\|_{\infty} /\|u\|_{\infty}, \quad N=180$ $u$ pt src, Nyström interp

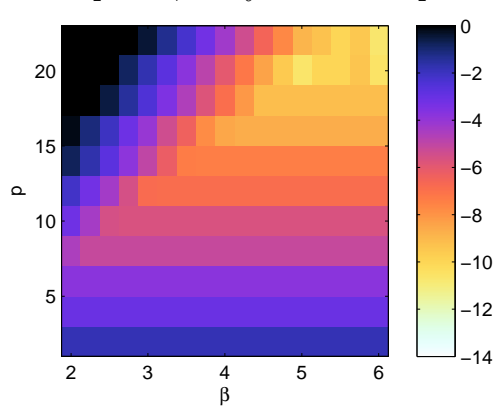

(c) $\log _{10}\|\hat{u}-u\|_{\infty} /\|u\|_{\infty}, \quad N=340$ $u$ pt src, trig poly interp

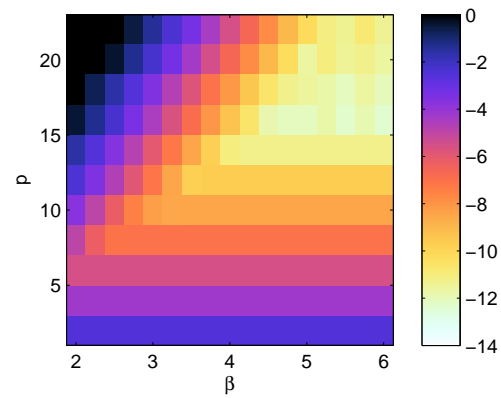

FIG. 3.1. Convergence of maximum relative $L^{\infty}(\Omega)$ error with respect to both parameters $p$ and $\beta$, at fixed $N$ and $\delta$, for the surrogate evaluation scheme of the double-layer potential solving a Laplace BVP in the domain of Fig. 2.2. (a) Dirichlet data $u(x, y)=e^{y} \cos x$, with $N=130$, trigonometric interpolant. $(b) u(x, y)=$ $\log \|(x, y)-(1,0.5)\|$, with $N=180$, Nyström interpolant. (c) $u(x, y)=\log \|(x, y)-(1,0.5)\|$, with $N=340$, trigonometric interpolant.

It is clear that the effort to compute (3.3) as presented scales as $O(\beta)$, once $N, N_{B}$ and $p$ are fixed. Thus we wish to know the minimum $\beta$ needed, and rewrite the second term in (3.6) as

$$
C p \exp \left(-2 \pi \delta \beta+p \log \left(2 \pi \delta \beta \frac{\gamma e}{p}\right)\right)
$$

For instance, fixing $\delta=2.5$ and taking $\gamma \approx 1.7$, we may solve (via rootfinding) for the approximate $\beta$ required for convergence by assuming that $C$ is $O(1)$ then equating the exponential term in (3.10) to the desired error level, e.g. $10^{-14}$. The predicted results are: for $p=10, \beta=4.2$ is sufficient. This matches well the finding that $\beta=4.0$ was sufficient to give around 14-digit accuracy in the example of Fig. 2.2(b). For $p=20, \beta=5.9$ is sufficient, indicating that $\beta$ need grow only weakly with $p$.

REMARK 11 (box centers). (3.10) suggests that increasing $\delta$ (moving the centers towards the far edge of their boxes) might increase accuracy. In practice, however, we find that this does not help because both $R / \rho$ and $\gamma$ increase for the worst-case boxes.

3.2. Numerical performance and the effect of a nearby singularity. We now study in more detail performance of the surrogate method for the Laplace double-layer potential, in the context of solving a Dirichlet BVP using the Nyström method.

We first return to the case presented in Fig. 2.2: boundary data $u(x, y)=e^{y} \cos x$, an entire function. We fix $N=130$ nodes (around the value at which the Nyström method has completely converged), and use a trigonometric polynomial interpolant to get $\tau$ at the fine nodes. With the $N_{B}$ boxes fixed, we consider convergence with respect to $p$ and $\beta$. Fig. 3.1(a) shows the resulting $L^{\infty}$ errors (estimated on a spatial grid of spacing 0.02) relative to $\|u\|_{\infty}$. The two terms in Theorem 10 are clearly visible: errors are always large at small $p$ (due to the first term in (3.6)), but even at large $p$ errors are large when $\beta$ is too small (due to the second term). The $\beta$ needed for convergence grows roughly linearly in $p$, as one would expect if the value of the log in (3.10) is treated as roughly constant. In both directions convergence appears exponential, exceeding 13 digits once $p=10$ and $\beta \geq 4$.

REMARK 12. For $p \geq 16$ the best achievable error (once $\beta$ has converged) worsens slightly as $p$ grows. We believe that this is due to catastrophic cancellation in the oscillatory integrand (3.2) for large $m$, combined with the usual double-precision round-off error. This seems to be a fundamental 
limit of the local expansion surrogate method implemented in floating-point arithmetic. However, the loss is quite mild: even at $p=22$ it is only 2.5 digits.

We now change to boundary data $u(x, y)=\log \|(x, y)-(1,0.5)\|$, which is still a Laplace solution in $\Omega$ and is still real analytic on $\partial \Omega$, but whose analytic continuation outside $\Omega$ has a singularity at the exterior point $(1,0.5)$, a distance of only 0.24 from $\partial \Omega$. Its preimage has distance from the real axis $\alpha_{*}:=-\operatorname{Im} Z^{-1}(1+0.5 i) \approx 0.176$. The Nyström method fully converged by $N=180$, as assessed by the error at a distant interior point and by the density values at the nodes $\tau_{j}$ : see the first two curves in Fig. 3.2(a). In fact the convergence rate matches $e^{-\alpha_{*} N}$, to be expected, since the Nyström convergence rate is known to be the same as the underlying quadrature scheme (see discussion after [24, Cor. 12.9]), which here is controlled by the singularity via the Davis theorem.

However, turning to surrogate evaluation, Fig. 3.1(b) shows that the error due to Taylor truncation converges quite slowly with $p$, as is inevitable for a nearby singularity, pushing the optimal $p$ up to around 22. In the best case only 10 digits are achieved. To evaluate $\tau$ at the fine nodes, the Nyström interpolant [24, Sec. 12.2] was used here rather than the trigonometric polynomial interpolant, for the following reason.

REMARK 13 (interpolants). In the regime where a nearby singularity in the right-hand side data controls the convergence rate (rather than the kernel function), the Nyström interpolant converges twice as fast as the trigonometric polynomial interpolant, as shown by Fig. 3.2(a). This reflects the fact that periodic trapezoid quadrature is "twice as good" as trigonometric interpolation [24, p.201], because the former is exact for Fourier components with index magnitudes up to $N-1$, but the latter is exact only up to N/2. Informally speaking, the trapezoid rule (and hence the Nyström interpolant) "beats the Nyquist sampling theorem by a factor of two!"

Thus the Nyström interpolant is preferred in this context when it is desired that $N$ be its smallest converged value. Fig. 3.2(b) and (c) show the loss in accuracy (and spurious evanescent waves which appear near $\partial \Omega$ ) that result from attempting to use the inferior trigonometric interpolant. Note that this loss would occur for any accurate close-evaluation scheme, since it is a loss of accuracy in the function $\tau$ itself. Nor is the Nyström interpolant perfect: it requires applying a $M$-by- $N$ dense matrix, and it appears to cause up to 1 digit more roundoff error than the trigonometic case.

However, by nearly doubling $N$ to 340 (somewhat wasteful), the Fourier coefficents of $\tilde{\tau}$ decay to machine precision by index $N / 2$ (see Fig. 3.2(a)) making the trigonometric interpolant as accurate as the Nyström one, whilst box radii decrease so that the $p$-convergence is faster. We show convergence for this trigonometric case in Fig. 3.1(c): 12 digits accuracy result at $p=16$ and $\beta=5$.

3.3. The Laplace single-layer case and a Neumann problem. Recall that the singlelayer potential is the real part of $v$ given by (2.10). By writing $\log 1 /(y-z)=\log 1 /\left(y-z_{0}\right)-$ $\log \left(1-\frac{z-z_{0}}{y-y_{0}}\right)$, and using the Taylor series for the second logarithm, we find that the single-layer version of (3.2) is

$$
c_{0}=\frac{1}{2 \pi} \int_{\partial \Omega}\left(\log \frac{1}{y-z_{0}}\right) \sigma(y)|d y|, \quad c_{m}=\frac{1}{2 \pi m} \int_{\partial \Omega} \frac{\sigma(y)}{\left(y-z_{0}\right)^{m}}|d y|, \quad m=1,2, \ldots
$$

The convergence of the surrogate scheme is then as least as good as for the double-layer case.

THEOREM 14. The version of Theorem 10 for the single-layer potential holds. That is, with the same conditions, the uniform error bound (3.6) holds, but with $\tau$ changed to $\sigma$, the potential $v$ given by (2.10), and $\hat{c}_{m}$ given by the $M$-node periodic trapezoid rule applied to the parametrized version of (3.11). 


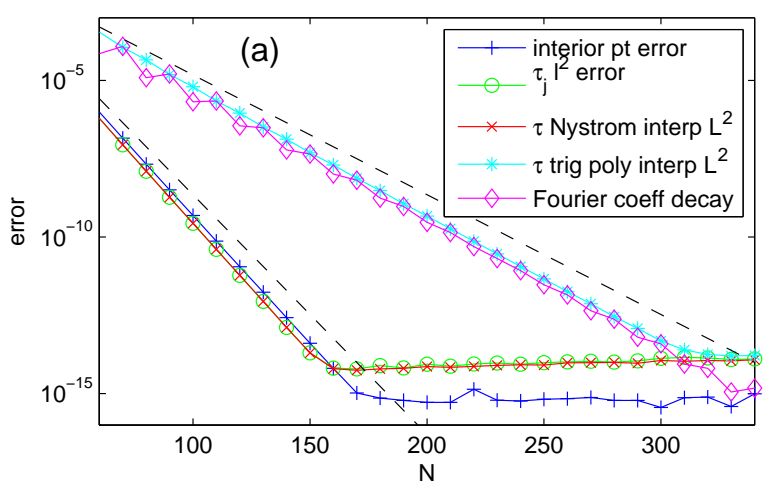

(b) $\log _{10}\|\hat{u}-u\|_{\infty} /\|u\|_{\infty}$ Nyström interp

(c) $\log _{10}\|\hat{u}-u\|_{\infty} /\|u\|_{\infty}$ trig interp

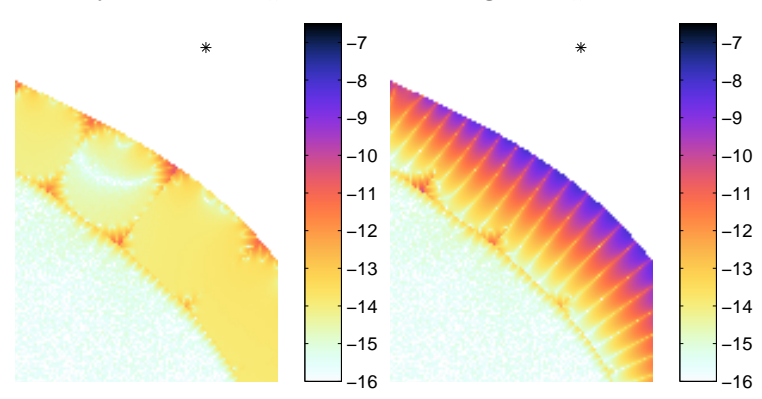

FIG. 3.2. Comparing two interpolants in the Nyström solution of $\tau$, for solution of the Dirichlet BVP for Laplace's equation, with data $u(x, y)=\log \|(x, y)-(1,0.5)\|$, in the domain of Fig. 2.2. (a) shows convergence of various errors: the solution at a distant interior point, the $l_{2}$ error at the nodes, the $L^{2}([0,2 \pi))$ errors for the Nyström and trigonometric interpolants, and the size of the N/2 Fourier coefficient of $\tau$. Dotted lines show exponential decay at the rates $e^{-\alpha_{*} N}$ and $e^{-\alpha_{*} N / 2}$. Fixing $N=180$, a zoomed plot of the relative error in the surrogate scheme is shown using (b) Nyström interpolant, and (c) trigonometric interpolant, near the singularity (shown by $a *$ ).

Proof. Using (3.11) and the Davis theorem, the coefficient errors analogous to (3.8) are

$$
\left|\hat{c}_{0}-c_{0}\right| \leq C\left|\log d\left(\Gamma_{\alpha}, z_{0}\right)\right| \frac{1}{e^{\alpha M}-1}, \quad\left|\hat{c}_{m}-c_{m}\right| \leq \frac{C}{m d\left(\Gamma_{\alpha}, z_{0}\right)^{m}} \frac{1}{e^{\alpha M}-1}, \quad m=1,2, \ldots
$$

But for each $m=0,1, \ldots$, this error is smaller than that in (3.8), after possibly a change in the constant $C$. The rest of the proof follows through. $\square$

REMARK 15. Note that the change in constant $C$ referred to is generally in the favorable direction: since $d\left(\Gamma_{\alpha}, z_{0}\right)<R$, the constant may be multiplied by a factor given by the larger of $R$ and $R|\log R|$, which are usually small. Also note that, since factors of $1 / m$ arise in the single-layer case, but were not taken advantage of, the p-convergence could probably be improved slightly.

As an application, we now report numerical results for the interior Laplace Neumann BVP

$$
\begin{aligned}
\Delta u & =0 & & \text { in } \Omega \\
\partial u / \partial n & =f & & \text { on } \partial \Omega
\end{aligned}
$$

which has a solution only if $f$ has zero mean on $\partial \Omega$, and in that case the solution is unique only up to an additive constant. Following [1, Sec. 7.2$]$ we use the integral equation

$$
\left(D^{*}+K+\frac{1}{2} I\right) \sigma=f,
$$

where $D^{*}$ has kernel $k(x, y)=\partial \Phi(x, y) / \partial n(x)$, and $K$ is the boundary operator which returns the value of its operand at some (fixed but arbitrary) point on $\partial \Omega$. The solution is then recovered up to an unknown constant by (2.9); we compare against the exact interior solution after subtracting the value of the constant measured at a single point.

As with the Dirichlet case, we test with boundary data coming from the entire function $u(x, y)=e^{y} \cos x$, or from the function with a nearby singularity $u(x, y)=\log \|(x, y)-(1,0.5)\|$. In the entire case, we find, as for the Dirichlet case, that Nyström convergence saturates at around $N=130$. Surrogate expansion (using the Nyström interpolant) then gives a maximum 
(a) $\log _{10}\left|u^{(N)}-u\right| /\|u\|_{\infty} \quad$ ext BVP, $u$ pt src

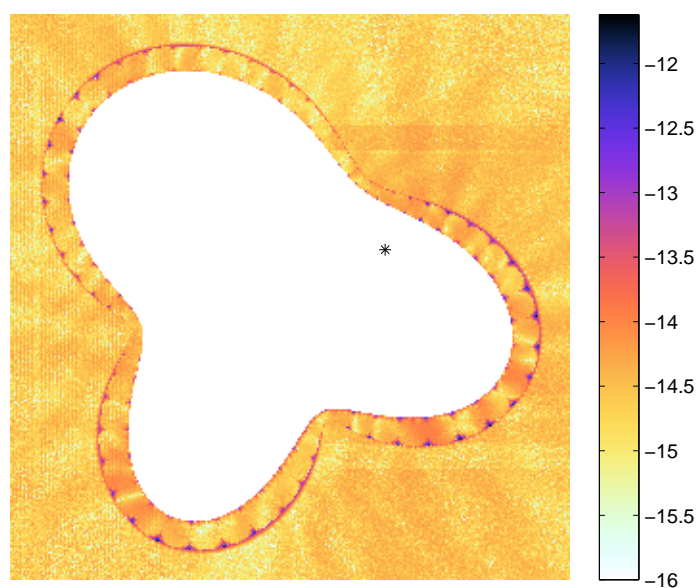

(b) $\log _{10}\|\hat{u}-u\|_{\infty} /\|u\|_{\infty}, \quad N=340$ $u$ pt src, trig poly interp

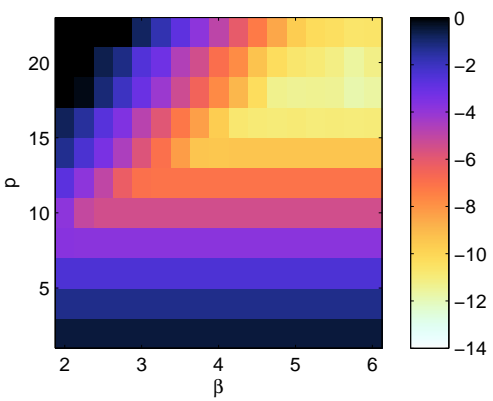

FIG. 4.1. Relative error for the surrogate scheme for the exterior Helmholtz Dirichlet problem at $\omega=30$ (diameter is 12 wavelengths), with known solution $\Phi\left(\cdot, x_{0}\right)$ with $x_{0}$ as shown by the * symbol. (a) error plot for $N=340, p=18$ and $\beta=6$ (many boxes are apparent). (b) $L^{\infty}(\Omega)$ error convergence with respect to parameters $p$ and $\beta$, at fixed $N=340$.

relative error of $6 \times 10^{-15}$ at $p=10$ and $\beta=4 .{ }^{4}$ For the singularity case, Nyström convergence completes at around $N=200$ and the maximum relative surrogate evaluation error is then found to be $6 \times 10^{-13}$ at $p=24$ and $\beta=5.5$. Notice that, for both data types, these single-layer errors are at least one digit improved over the double-layer errors reported in Sec. 3.2; this may be explained by Remark 15. Indeed, the convergence plots analogous to Fig. 3.1(a) and (b) are very similar but gain around 1 extra digit of accuracy.

4. The Helmholtz equation and an $O(N)$ close evaluation scheme. We now move to a PDE for which we no longer have theorems, but which has important applications.

4.1. Implementation and convergence test for the Helmholtz equation. The above close evaluation scheme for $\omega=0$ with real-valued potentials is very easily adapted to the Helmholtz equation $(\omega>0)$ with complex potentials, by replacing a couple of formulae. The $p$-term Taylor expansion (3.4) for $v$ (whose real part was taken to get $u$ ), is replaced by a local (Fourier-Bessel) expansion with $(2 p-1)$ terms,

$$
\hat{u}(z)=\sum_{|m|<p} c_{m} e^{i m \theta} J_{m}(\omega r), \quad \text { where } z-z_{0}=r e^{i \theta}, \quad z \text { in box } B,
$$

i.e. $(r, \theta)$ is the polar coordinate system with origin $z_{0}$. For the single-layer potential, recalling (1.3) and the Graf addition formula $[29,(10.3 .7)]$, the formula (3.11) is replaced by

$$
c_{m}=\frac{i}{4} \int_{\partial \Omega} e^{-i m \theta_{y}} H_{m}^{(1)}\left(\omega r_{y}\right) \sigma(y) d s_{y}, \quad|m|<p
$$

where $\left(r_{y}, \theta_{y}\right)$ are the polar coordinates of the point $y$ relative to the origin $z_{0}$. Using the addition formula, the reflection formulae, after some simplification, the Cauchy formula (3.2) is replaced

\footnotetext{
${ }^{4}$ Curiously, the trigonometric interpolant in this case does not become fully accurate until $N=340$, which is much more than the $N=130$ for the Dirichlet case. We cannot explain this-neither result is as predicted by Remark 13-and it tells us that there is still more to understand about the Nyström method convergence rates in analytic BVP settings.
} 
by

$$
c_{m}=\frac{i \omega}{8} \int_{\partial \Omega}\left[e^{-i(m-1) \theta_{y}-i \nu_{y}} H_{m-1}^{(1)}\left(\omega r_{y}\right)-e^{-i(m+1) \theta_{y}+i \nu_{y}} H_{m+1}^{(1)}\left(\omega r_{y}\right)\right] \tau(y) d s_{y}, \quad|m|<p,
$$

where $\nu_{y}$ is the angle of the outward normal at $y \in \partial \Omega$. The above three formulae are all the changes needed for the Helmholtz version of the scheme.

We have already seen in Fig. 1.1(d) that the Laplace predictions for the native evaluation error (theorems 3 and 8) also hold well for the low-frequency Helmholtz equation. This is to be expected, since for $\omega>0$ the fundamental solution (1.3) remains analytic away from the origin, so the Davis theorem applies to the parametrized (1.5) and (2.9), allowing the Laplace convergence rate to be approached. (We leave the Helmholtz equivalents of the tight theorems 3 and 8 for future work.) Therefore we apply the same criterion for the "bad strip" $\Omega_{\text {bad }}$ as in section 3 .

We test the scheme in the context of BVP applications, namely the Dirichlet problem in an exterior domain $\Omega$, with the usual Sommerfeld radiation condition [10, (3.62)], for which the integral equation (1.4) is replaced by the so-called combined-field formulation [10, p. 48]

$$
\left(D-i \omega S+\frac{1}{2} I\right) \tau=f
$$

which is well conditioned for all $\omega>0$. Here the potential is represented as

$$
u(x)=\int_{\partial \Omega}\left[\frac{\partial \Phi(x, y)}{\partial n(y)}-i \omega \Phi(x, y)\right] \tau(y) d s_{y}, \quad x \in \Omega
$$

and we choose the right-hand side data as coming from an interior point-source shown by the * in Fig. 4.1(a). To achieve spectral accuracy in the Nyström method, special quadratures for the weak logarithmic singularity are needed; we use the scheme of Kress [23] (also see [16]).

We fix $\omega=30$ (thus $\partial \Omega$ is 12 wavelengths across), and find that the Nyström method converges at around $N=340$. A maximum relative surrogate evaluation error around $3 \times 10^{-12}$ then is achieved at $p=18, \beta=6$, as shown in Fig. 4.1(a). This is dominated by errors in the box corners furthest from $\partial \Omega$, especially those with distortion due to a convex part of $\partial \Omega$. Since this holds largely independently of the singularity location $x_{0}$, we believe it is instead controlled by the wavenumber $\omega$. The $L^{\infty}(\Omega)$ convergence with $p$ and $\beta$ is very similar to the Laplace case, as shown in Fig. 4.1(b). $p=18$ is close to optimal, since for $p>20$ the error worsens - the culprit is believed to be as in Remark (12), but demands further study. However, simply by making the boxes slightly narrower and more numerous by setting $N_{B}=\lceil N / 4\rceil$, with $25 \%$ more effort we cut maximum relative error to $3 \times 10^{-13}$.

REMARK 16 (interpolants revisited). A natural question is: does Remark 13 hold for the Helmholtz equation? The answer is no, at least for the Kress scheme (which is one of the best known [16]). Although a Nyström interpolant does exist for the weakly-singular kernels [23, (3.3)], we believe it has little advantage over the trigonometric interpolant. This is because the productquadrature scheme of Kress is only accurate for Fourier components of indices up to N/2. In data with a nearby singularity with preimage a distance $\alpha_{*}$ from the real axis, the convergence rate is thus only $e^{-\alpha_{s} N / 2}$, or half that of the Laplace case.

4.2. Evaluation via a correction to the fast multipole method. At higher frequencies $\omega$ and/or complex geometries $\partial \Omega$, the $N$ needed for Nyström convergence is pushed higher. For instance, with fixed geometry the high frequency requirement is $N=O(\omega)$, i.e. a constant number of nodes per wavelength [3, 17]. The surrogate scheme as presented requires $O\left(p N^{2}\right)$ effort, by evaluating $p$ coefficients at $N_{B}=O(N)$ centers using $M=O(N)$ fine node kernel evaluations for 
each. Clearly a better scaling with $N$ is preferred. We now show that at fixed frequency, linear scaling is easy to achieve by locally correcting the FMM.

Fixing a box $B$ with center $z_{0}$, and taking e.g. the Helmholtz single-layer potential (2.9), we split the integral into "near" and "far" parts using a cut-off radius $G$. We apply the surrogate local expansion only for the near part to get

$$
\hat{u}(x)=\sum_{|m|<p} c_{m}^{\text {(near) }} e^{i m \theta} J_{m}(\omega r)+\int_{y \in \partial \Omega,\left|y-z_{0}\right|>G} \Phi(x, y) \sigma(y) d s_{y}, \quad x \text { in box } B,
$$

where, adapting (4.1), the coefficients of the potential due to the near part of the integral only are,

$c_{m}^{(\text {near })}=\frac{i}{4} \int_{y \in \partial \Omega,\left|y-z_{0}\right| \leq G} e^{-i m \theta_{y}} H_{m}^{(1)}\left(\omega r_{y}\right) \sigma(y) d s_{y} \approx \frac{i \pi}{2 M} \sum_{j \in J_{\text {near }}} e^{-i m \theta_{Z\left(s_{j}\right)}} H_{m}^{(1)}\left(\omega r_{Z\left(s_{j}\right)}\right) \tilde{\sigma}\left(s_{j}\right) Z^{\prime}\left(s_{j}\right)$,

and where $J_{\text {near }}:=\left\{j:\left|Z\left(s_{j}\right)-z_{0}\right| \leq G\right\}$ is the index set of the "near" subset of the fine nodes $\left\{s_{j}\right\}_{j=1}^{M}$. The cut-off $G$ must be large enough that the fictitious singularities induced at the ends of the near interval are distant enough not to slow down the convergence of the local expansion, thus we choose $G$ several times the box radius $R$, and so evaluating these sums takes $O(p)$ effort. Applying the fine quadrature to the far part of the integral in (4.5) gives

$$
\int_{y \in \partial \Omega,\left|y-z_{0}\right|>G} \Phi(x, y) \sigma(y) d s_{y} \approx \frac{2 \pi}{M} \sum_{j=1}^{M} \Phi\left(x, Z\left(s_{j}\right)\right) \tilde{\sigma}\left(s_{j}\right) Z^{\prime}\left(s_{j}\right)-\frac{2 \pi}{M} \sum_{j \in J_{\text {near }}} \Phi\left(x, Z\left(s_{j}\right)\right) \tilde{\sigma}\left(s_{j}\right) Z^{\prime}\left(s_{j}\right) .
$$

The first sum can be evaluated for all targets $x$ in all boxes in a single FMM call. Making the reasonable assumption that the user demands $O(1)$ targets per box, this FMM call requires $O(N)$ effort. Finally, the second sum is a local correction that takes $O(1)$ effort. The total effort to evaluate $\hat{u}(x)$ for all $x$ in all $N_{B}$ boxes is thus $O(p N)$. A similar scheme applies for the double-layer potential.

Since the first (FMM) term in (4.7) accurately approximates $u$ everywhere except in a narrower strip $A_{\alpha}$ with $\alpha=10 \pi / \beta N$, we in fact may, and will, shrink the boxes in the normal direction, as long as they cover $A_{\alpha}$, whilst using this first term in the remaining part of $\Omega_{\text {bad }}$. This has the advantage of avoiding larger errors that tend to occur in the distant corners of boxes.

REMARK 17 (no end corrections). At this point the reader might very well suspect that, since both of the above integrals are on non-periodic intervals, the trapezoid rule would give at best low-order $O(1 / M)$ convergence unless higher-order end correction rules were used. In fact, spectral accuracy equal to that of the trapezoid rule on the original periodic integrand is observed. The reason is slightly subtle: the endpoint errors in the two integrals cancel, since their sum ultimately represents to high order the trapezoid rule applied to the periodic analytic integrand in (2.9). Thus our simple splitting achieves spectral accuracy with neither a partition of unity nor end-point quadrature corrections.

4.3. High frequency scattering example. We now detail the application of the $O(N)$ method just described to a high frequency scattering problem with Dirichlet boundary condition; this corresponds to an acoustically sound-soft obstacle. We choose a complicated (but analytic) boundary $\partial \Omega$ given by the polar Fourier series $f(\theta)=1+\sum_{n=1}^{40} a_{n} \cos (n \theta)+b_{n} \sin (n \theta)$, with $a_{n}$, $b_{n}$ uniform random in $[-0.04,0.04]$, and parametrized by $\theta$; see Fig. $4.2(\mathrm{a})$. We choose $\omega=250$ such that the obstacle is 100 wavelengths across. For the scattering problem with plane wave $u_{\text {inc }}(x)=e^{i \omega \hat{d} \cdot x}$ incident at angle $\hat{d}=(\cos -\pi / 5, \sin -\pi / 5)$, the total potential (physical field) is 
(a) $u_{\text {tot }}$
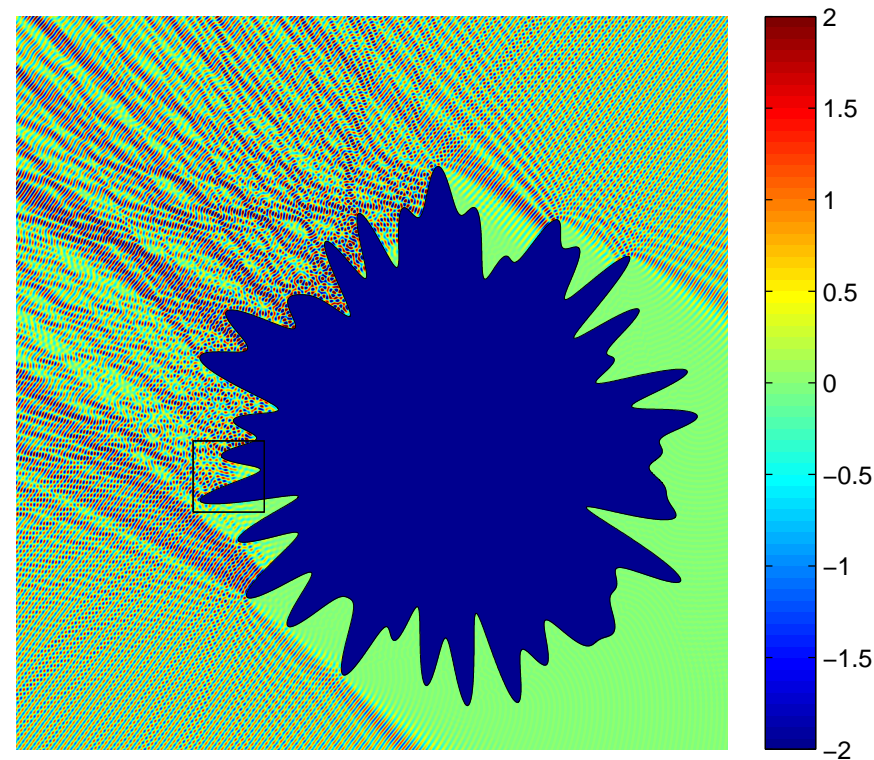

(c) $\log _{10}\left|u^{(N)}-u\right| /\left\|u_{\text {tot }}\right\|_{\infty}$

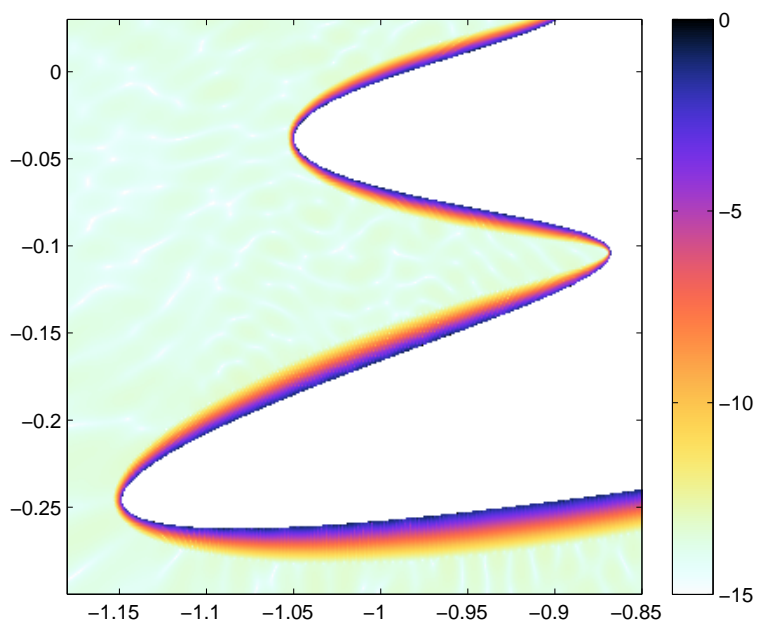

(b) $u_{\text {tot }}$ zoomed

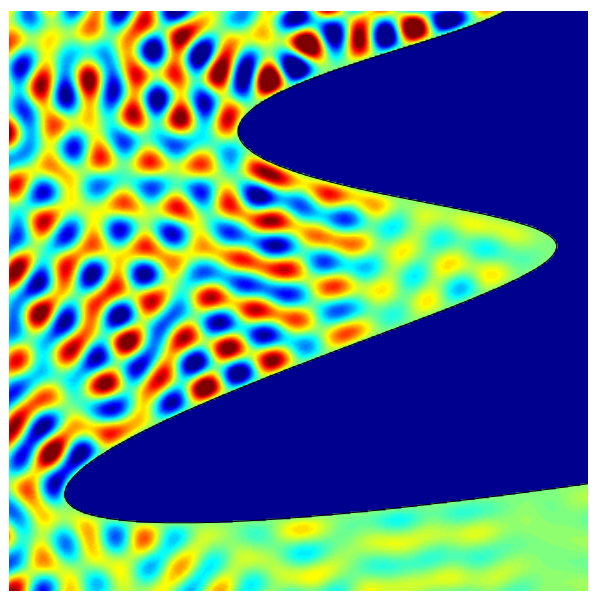

(d) $\log _{10}|\hat{u}-u| /\left\|u_{\text {tot }}\right\|_{\infty}$

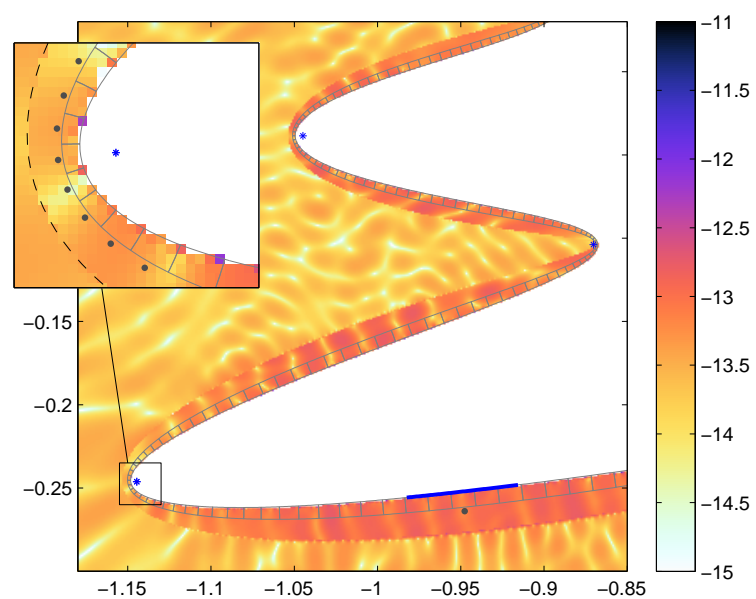

FIG. 4.2. High frequency sound-soft scattering example, $\omega=250$ (diameter is 100 wavelengths), needing $N=9000$ nodes. (a) Total field $u_{\text {tot }}=u_{\text {inc }}+u$. (b) Zoom of total field for black box shown in (a). (c) Relative error (relative to $\left\|u_{t o t}\right\|_{\infty}=6.2$ ) in native evaluation $u^{(N)}$ in same region as $(b)$. (d) Relative error in surrogate scheme $\hat{u}$ in same region as (b), with $p=26, \beta=6$. Boxes are shown in gray, and a single center $z_{0}$ (grey dot) with its corresponding "near" set of boundary points (thick blue line), and domain Schwarz singularities (*). Note the change in color scale between (c) and (d). The inset in (d) is a zoom of the highly convex region, and also shows all centers (grey dots) and $\Gamma_{\alpha_{b a d}}$ the boundary of $\Omega_{b a d}$ (dotted line).

$u_{\text {tot }}=u_{\text {inc }}+u$ where the (radiative) scattered potential $u$ solves the exterior Dirichlet problem with boundary data $f=-\left.u_{\mathrm{inc}}\right|_{\partial \Omega}$. For this BVP, as before, we solve the integral equation (4.3) then evaluate $u$ via (4.4).

We find that $N=9000$ is needed to get 13-digit Nyström convergence for $u$, as assessed 
at a variety of exterior points lying outside the "bad strip" $\Omega_{\mathrm{bad}}$. This averages 13 nodes per wavelength, although it is as little as 4.6 nodes per wavelength where $\left|Z^{\prime}(s)\right|$ is largest. It takes 73 s to fill the dense $N \times N$ matrix, and a further $14 \mathrm{~s}$ to solve the system via GMRES using dense matrix-vector products (needing 95 iterations to exceed a relative residual of $10^{-12}$ ).

REMARK 18. Here all timings are reported for a System76 laptop with $2.6 \mathrm{GHz}$ Intel i73720QM CPU, 16GB of RAM, running MATLAB 2012b [32], MPSpack version 1.32 [4], and FMMLIB2D version 1.2 [15]. We use MATLAB's native Bessel functions. For Hankel functions in (4.6) and the second sum in (4.7) (and their double-layer analogs) we use a MEX interface to hank103.f [15] for $m=0,1$, and upwards recurrence [29, (10.6.1)]. Most operations use only a single core; the only which exploit all four cores are FMMLIB2D and the matrix-vector products in GMRES.

We fix a set of around $8 \times 10^{6}$ evaluation points, namely those on a $3300 \times 3400$ grid of spacing $10^{-3}$ which lie in the exterior of $\partial \Omega$; see Fig. 4.2. Evaluation of $u$ at these targets using the $N$ native nodes and the FMM took $24 \mathrm{~s}$, and gives the relative errors in Fig. 4.2(c): large errors are apparent near $\partial \Omega$. We now apply the surrogate close evaluation to the around $2.2 \times 10^{5}$ points lying in $\Omega_{\text {bad }}$. We set $N_{B}=\lceil N / 3\rceil=3000$, rather more than the $\lceil N / 5\rceil$ recommended before: this helps reduce errors by shrinking the box radii $R$. We found convergence at around $p=26$, $\beta=6$ (this high $p$ is needed because boxes are up to 0.7 wavelengths in size). A cut-off radius $G$ that does not induce additional error was found to be 1.5 times the maximum width of the bad strip in the surrounding 7 boxes; this gives between 84 and 326 "near" fine points, but with a mean of only 107 (note that this is less than the 288 that would be required for three 16-node panels and the same $\beta$; see remark 21). With the above parameters we achieve a maximum error in $u$ of $6 \times 10^{-12}$, in $28 \mathrm{~s}$ computation time. Much of this is spent on direct sums in (4.6) and (4.7) (the fine FMM in (4.7) takes only $1.5 \mathrm{~s}$ ), as well as geometric and inevitable MATLAB overheads. We found this to be 50 times faster than directly applying the $O\left(N^{2}\right)$ formulae from section 4.1. Fig. 4.2(d) plots a zoom of the resulting relative error.

REMARK 19 (Reference solution). In previous examples u was analytically known. In order to assess the error in $\hat{u}$ for this example we compute a reference $u$ in the following expensive fashion. Outside $\Omega_{\text {bad }}$ we used the FMM from the density $\left\{\tau_{j}\right\}_{j=1}^{N}$ refined by a factor 10 by trigonometric interpolation. Inside $\Omega_{\text {bad }}$ we did the same but with a factor $10^{3}$, apart from the few hundred points in the narrow strip inside $\Gamma_{10^{-3} \alpha_{\text {bad }}}$. For these last points we used 9th-order polynomial extrapolation from points distances $\left\{2^{j}\left(10^{-2} \pi / N\right)\left|Z^{\prime}(s)\right|\right\}_{j=0}^{9}$ along the normal direction. This appears to give around 12 digits; we are not able reliably to get more.

REMARK 20 (Convex vs concave). The largest errors occur at the corners of boxes near highly-convex parts of the boundary; we believe that this is due to the nearby interior Schwarz singularity of $\partial \Omega$, which generically induces a singularity in the Helmholtz continuation of the solution u [27] (briefly reviewed in [3, Sec. 3.1]). The latter in turn slows the p-convergence of the expansion. By contrast, concave parts have Schwarz singularities on the physical side of $\partial \Omega$ which thus do not affect $u$. We believe this explains why lower accuracy is reported at convex (but not concave) locations in $Q B X$ [21].

5. Conclusion and discussion. Firstly, we analyzed the spatial distribution of the error in evaluating Laplace layer potentials using the popular global periodic trapezoid rule. The key tools were generalizations of the Davis theorem, and the annular conformal map between the complex parameter plane and the physical plane. We found (Theorems 3 and 8) that the exponential convergence rate at a point is simply the imaginary part of its preimage under this map, a result believed to be new. Error contours are thus given by "imaginary parameter translations" of the boundary; these sweep out a strip $\Omega_{\text {bad }}$ where errors are unacceptable. Empirically, errors are similar in the Helmholtz case.

Secondly, we devised a surrogate local expansion method for accurate evaluation in $\Omega_{\mathrm{bad}}$. Our 
main analytical result is its exponential convergence (and hence that of QBX [21]) in the analytic Laplace case (Theorems 10 and 14). The scheme can be executed via the FMM in $O(N)$ time, and generalizes easily to the Helmholtz equation, as we showed in a challenging high-frequency scattering application. Our scheme gives errors close to machine precision, given density values at only the number $N$ of nodes sufficient for the Nyström method to converge. Our experiments also highlighted the need for more understanding of the Nyström exponential convergence rate and of the situations in which the trigonometric interpolant is inferior to the Nyström one.

REMARK 21 (Adaptivity). We used global quadrature in this preliminary study; an adaptive panel-based (composite) underlying quadrature, however, would be preferred in a production code, and would allow refinement at corners. The surrogate scheme is easy to implement with Gaussian panels, needing only one Lagrange interpolation to the fine nodes. Furthermore, the fast scheme of section 4.2 becomes simpler: the only FMM needed is the native evaluation $u^{(N)}$, corrected in $O(N)$ effort by refining only the 3 nearest panels. Comparison against the recent panel-based scheme of Helsing [17] would be desired.

We expect the generalization to surfaces in $\mathbb{R}^{3}$ to be fruitful, both for close evaluation and for singular Nyström quadratures [21]. Since only smooth interpolation, the local expansion, and the addition theorem are needed, this should be simpler to implement than existing highorder schemes $[10,8,35,7]$. Analysis in $\mathbb{R}^{3}$ remains daunting: one can no longer exploit the link between Laplace solutions in $\mathbb{R}^{2}$ and holomorphic functions in $\mathbb{C}$. However, analysis for the Helmholtz case in $\mathbb{R}^{2}$, both of native errors and of the surrogate scheme, should be tractable via Vekua's map from holomorphic functions to Helmholtz solutions [34, 19, 6, 28].

Acknowledgments. The author is very grateful to Hanh Nguyen, via the support of the Women in Science Project at Dartmouth College, for testing a Laplace double-layer implementation in Spring of 2011. The author also benefited from discussions with Stephen Langdon, Zydrunas Gimbutas, Leslie Greengard, Andreas Klöckner, Mike O'Neil, and Nick Trefethen. This work is supported through the National Science Foundation via grants DMS-0811005 and DMS-1216656.

Appendix A. Code. While we have not yet released a formal package implementing the methods of this paper, we make available codes that generated the figures here:

http://math.dartmouth.edu/ ahb/software/closeeval

These require MATLAB [32], MPSpack version 1.32 [4], and FMMLIB2D version 1.2 [15].

\section{REFERENCES}

[1] K. Atkinson, The numerical solution of integral equations of the second kind, Cambridge University Press, 1997.

[2] K. Atkinson And Y.-M. Jeon, Algorithm 788: Automatic boundary integral equation programs for the planar Laplace equation, ACM Trans. Math. Software, 24 (1998), pp. 395-417.

[3] A. H. Barnett And T. Betcke, Stability and convergence of the Method of Fundamental Solutions for Helmholtz problems on analytic domains, J. Comput. Phys., 227 (2008), pp. 7003-7026.

[4] - MPSpack: A MATLAB toolbox to solve Helmholtz PDE, wave scattering, and eigenvalue problems, 2008-2012. http://code.google.com/p/mpspack/.

[5] A. H. Barnett and L. Greengard, A new integral representation for quasi-periodic fields and its application to two-dimensional band structure calculations, J. Comput. Phys., 229 (2010), pp. 6898-6914.

[6] T. Betcke, Computations of Eigenfunctions of Planar Regions, PhD thesis, Oxford University, UK, 2005.

[7] J. Bremer and Z. Gimbutas, A Nyström method for weakly singular integral operators on surfaces, J. Comput. Phys., 231 (2012), pp. 4885-4903.

[8] O. P. Bruno And L. A. Kunyansky, A fast, high-order algorithm for the solution of surface scattering problems: basic implementation, tests, and applications, J. Comput. Phys., 169 (2001), pp. 80-110.

[9] S. N. Chandler-Wilde, I. G. Graham, S. Langdon, and E. A. Spence, Numerical-asymptotic boundary integral methods in high-frequency acoustic scattering, Acta Numer., (2012), pp. 89-305. 
[10] D. Colton And R. Kress, Inverse acoustic and electromagnetic scattering theory, vol. 93 of Applied Mathematical Sciences, Springer-Verlag, Berlin, second ed., 1998.

[11] P. J. Davis, On the numerical integration of periodic analytic functions, in Proceedings of a Symposium on Numerical Approximations, R. E. Langer, ed., University of Wisconsin Press, 1959.

[12] P. J. Davis, The Schwarz function and its applications, The Mathematical Association of America, Buffalo, N. Y., 1974. The Carus Mathematical Monographs, No. 17.

[13] P. J. Davis and P. Rabinowitz, Methods of Numerical Integration, Academic Press, San Diego, 1984.

[14] S. D. GEDNEY, On deriving a locally corrected Nyström scheme from a quadrature sampled moment method, IEEE Trans. Antennas Propag., 51 (2003), pp. 2402-2412.

[15] Z. Gimbutas and L. Greengard, FMMLIB2D, Fortran libraries for fast multipole method in two dimensions, 2011. http://www.cims.nyu.edu/cmcl/fmm2dlib/fmm2dlib.html.

[16] S. Hao, A. H. Barnett, P. G. Martinsson, and P. Young, High-order accurate Nyström discretization of integral equations with weakly singular kernels on smooth curves in the plane, 2012. preprint, arxiv: 1112.6262 .

[17] J. Helsing, Solving integral equations on piecewise smooth boundaries using the RCIP method: a tutorial, 2012. preprint, 34 pages, arXiv:1207.6737v3.

[18] J. Helsing And R. Ojala, On the evaluation of layer potentials close to their sources, J. Comput. Phys., 227 (2008), pp. 2899-2921.

[19] P. Henrici, A survey of I. N. Vekua's theory of elliptic partial differential equations with analytic coefficients, Z. Angew. Math. Phys., 8 (1957), pp. 169-203.

[20] D. B. Hunter, The evaluation of integrals of periodic analytic functions, BIT Numer. Math., 11 (1971), pp. $175-180$

[21] A. Klöckner, A. H. Barnett, L. Greengard, and M. O'Neil, Quadrature by expansion: a new method for the evaluation of layer potentials, 2012. submitted, arxiv:1207.4461.

[22] J. KorevaAr, Book review of "The Schwarz function and its generalization to higher dimensions" by H. S. Shapiro, Bull. Amer. Math. Soc., 31 (1994), pp. 112-116.

[23] R. KREss, Boundary integral equations in time-harmonic acoustic scattering, Mathl. Comput. Modelling, 15 (1991), pp. 229-243.

[24] R. KREss, Linear Integral Equations, vol. 82 of Applied Mathematical Sciences, Springer, second ed., 1999.

[25] A. MAYO, Fast high order accurate solution of Laplace's equation on irregular regions, SIAM J. Sci. Stat. Comput., 6 (1985), pp. 144-157.

[26] A. McKenney, An adaptation of the fast multipole method for evaluating layer potentials in two dimensions, Computers Math. Applic., 31 (1996), pp. 33-57.

[27] R. F. MillaR, Singularities and the Rayleigh hypothesis for solutions to the Helmholtz equation, IMA J. Appl. Math., 37 (1986), pp. 155-171.

[28] A. Moiola, R. Hiptmair, and I. Perugia, Vekua theory for the Helmholtz operator, Z. Angew. Math. Phys., 62 (2011), pp. 779-807.

[29] F. W. J. Olver, D. W. Lozier, R. F. Boisvert, and C. W. Clark, eds., NiST Handbook of Mathematical Functions, Cambridge University Press, 2010. http://dlmf.nist.gov.

[30] C. Schwab And W. L. Wendland, On the extraction technique in boundary integral equations, Math. Comp., 68 (1999), pp. 91-122.

[31] E. M. Stein and R. Shakarchi, Complex Analysis (Princeton Lectures in Analysis, No. 2), Princeton University Press, 2003.

[32] The MathWorks, Inc., MATLAB software, Copyright (c) 1984-2012. http://www.mathworks.com/matlab.

[33] L. N. Trefethen, Approximation Theory and Approximation Practice, SIAM, 2012. http://www.maths.ox.ac.uk/chebfun/ATAP.

[34] I. N. Vekua, Novye metody rezhenija elliptickikh uravnenij, (OGIZ, Moscow and Leningrad, 1948); English translation: New methods for solving elliptic equations, North-Holland, 1967.

[35] L. Ying, G. BIRos, AND D. Zorin, A high-order 3D boundary integral equation solver for elliptic PDEs in smooth domains, J. Comput. Phys., 216 (2006), pp. 247-275. 